\title{
Mixed-strategy equilibria in the Nash Demand Game
}

\author{
David A. Malueg
}

Received: 26 February 2008 / Accepted: 2 June 2009 / Published online: 19 June 2009

(C) The Author(s) 2009. This article is published with open access at Springerlink.com

\begin{abstract}
In the Nash Demand Game, each of the two players announces the share he demands of an amount of money that may be split between them. If the demands can be satisfied, they are; otherwise, neither player receives any money. This game has many pure-strategy equilibria. This paper characterizes mixed-strategy equilibria. The condition critical for an equilibrium is that players' sets of possible demands be balanced. Two sets of demands are balanced if each demand in one set can be matched with a demand in the other set such that they sum to one. For Nash's original game, a complete characterization is given of the equilibria in which both players' expected payoffs are strictly positive. The findings are applied to the private provision of a discrete public good.
\end{abstract}

Keywords Nash Demand Game · Divide-the-Dollar Game · Mixed-strategy equilibria

JEL Classification $\quad \mathrm{C} 72 \cdot \mathrm{C} 78$

\section{Introduction}

This paper characterizes equilibria in the stylized bargaining model known as the Nash Demand Game. In the Nash Demand Game, two players have the opportunity to divide an amount of money between them. For simplicity, this amount is taken to

The author thanks three anonymous referees for their comments and suggestions.

D. A. Malueg $(\varangle)$

Department of Economics, University of California Riverside,

3136 Sproul Hall, Riverside, CA 92521, USA

e-mail: david.malueg@ucr.edu 
be one dollar. ${ }^{1}$ Players simultaneously announce their demanded shares of the money. If both demands can be satisfied, then they are; otherwise neither player receives any money. A player's payoff is the amount of money he receives. ${ }^{2}$

There are many pure-strategy equilibria to Nash Demand Game. In particular, for any $s \in[0,1]$, it is an equilibrium for one player to demand $s$ and the other to demand $1-s$. Such an equilibrium fully distributes the dollar to the players. The only purestrategy equilibrium (in undominated strategies) yielding disagreement has each player demand 1. Such disagreement relies on implausible individual demands and does not match the observations of disagreement in experiments. And unrealistically, pure-strategy equilibria predict disagreement occurs for sure or not at all. Of course, in the real world, labor strikes sometimes occur and court cases do not always settle before trial. Similarly, experimental settings of bargaining games find occasional disagreement.

Over two decades ago, Roth (1985) proposed a focal-point explanation for disagreement in bargaining, arguing that disagreement might naturally arise in the play of a mixed-strategy equilibrium. In Roth's experiments, the Nash Demand Game appeared to have two focal points, and Roth suggested equilibrium disagreement arose as a failure of the players to coordinate on one of these two pure-strategy equilibria. In particular, he provided a mixed-strategy equilibrium in which each player randomizes over two distinct demands-and one pair of announced demands more than exhausts the dollar, resulting in disagreement. Later experimenters also investigated the consequences of multiple focal points in the Nash Demand Game [for surveys see Roth (1995) and Camerer (2003, Ch. 4)].

The current paper addresses the following question raised by Roth (1985) analysis: exactly what are the (mixed-strategy) equilibria in the Nash Demand Game? A key condition is identified: for given equilibrium (mixed) strategies, players' sets of possible demands must be balanced. Players' sets of possible demands are balanced if each demand, $s$, in one player's set of possible demands can be matched with the complementary demand, $1-s$, in the other player's set of possible demands. Conversely, if the players' payoffs are also continuous over the region of agreement, then (almost) any pair of balanced sets of demands can be a part of an equilibrium in which players randomize completely over their sets of possible demands. This second result requires approximating closed sets of possible demands by finite subsets, and then looking at the limiting case as the finite subsets become dense in the original sets of demands. Because payoffs are discontinuous (where demands just sum to 1), techniques used are reminiscent of those used by Dasgupta and Maskin (1986) to establish existence of equilibrium in games with discontinuous payoffs. However, here existence of equilibrium is not an issue-there are many pure-strategy equilibria. Rather, the goal is to derive properties of the limiting case from equilibria in the finite

\footnotetext{
${ }^{1}$ For this reason, the game has also been called the Divide-the-Dollar Game.

2 The Nash Demand Game can also be given an exchange interpretation. Suppose a seller has a product worth nothing to her but worth 1 to the prospective buyer. These values are common knowledge to the buyer and seller. Simultaneously the seller announces the least price, $p_{S}$, she is willing to accept for the product, and the buyer announces the greatest price, $p_{B}$, he is willing to pay for the product. If $p_{S}>p_{B}$, no trade occurs. If $p_{S} \leq p_{B}$, then exchange takes place at a price $p \in\left[p_{S}, p_{B}\right]$ and the payoff to the seller is $p$, to the buyer $1-p$.
} 
approximations. In this respect, this paper is closer in spirit, for example, to Baye et al. (1996a,b) who study the mixed-strategy equilibrium of a continuous strategy-space game as the limit of games with finite strategy sets, thereby deducing properties of the limiting equilibrium from properties of the finite games. Finally, Nash's original formulation has each player receive the amount he demands if demands are compatible, with a player's utility just being the amount of money received. For this formulation, a complete characterization is given of the equilibria in which both players' expected payoffs are strictly positive. Harsanyi (1973) showed mixed-strategy equilibria of a full-information game may be viewed as limits of equilibria in nearby games with private information. ${ }^{3}$ Thus, the range of mixed-strategy equilibria derived also points to the richness of equilibria that might be found by incorporating private information into the Nash Demand Game.

The Nash Demand Game has been a workhorse in bargaining theory. Among the efficient pure-strategy equilibria in the Nash Demand Game, symmetry identifies the outcome in which each player demands 1/2. Much of the literature, beginning with Nash $(1950,1953)$, has provided additional rationales for this selection, also known as the Nash bargaining solution. ${ }^{4}$ While these approaches explain the particular equilibrium outcome in which players evenly divide the available money, they do not explain occasions of disagreement.

Crawford (1982) proposed a two-stage model where disagreement can occur. In the first stage, players choose to be "cooperative" (not announcing a demand) or to announce demands. Then in the second stage demands are observed; a player who has announced a demand has the option of "backing down" at some cost, ${ }^{5}$ which is not revealed until the second stage. In this model, equilibrium disagreement can occur when players announce incompatible demands and then find that they would incur high costs of backing down. Ellingsen and Miettinen (2008) take an approach similar to Crawford's by introducing a known cost of commitment but assuming the commitment "sticks" only with some probability less than 1. Equilibrium disagreement can arise here, too, but only when each player has demanded 1 for himself.

Section 2 below introduces a generalized Nash Demand Game. Section 3 characterizes equilibria in which both players gain from bargaining, relative to disagreement.

\footnotetext{
3 See Radner and Rosenthal (1982), Milgrom and Weber (1985), Kahn and Sun (1995), and Fu (2008) for the development of the related point that mixed-strategy equilibria in games with private information may be "purified," that is, they can be represented by equivalent equilibria in pure strategies.

4 Beyond the Nash (1950) axiomatization, Nash (1953) also presented a "smoothed" game in which the probability that demands are met decreases rapidly to zero as the sum of demands exceeds 1 . Letting the "amount of smoothing" go to zero, Nash finds the equal division as the limit of the unique equilibrium outcomes in the smoothed games (see also Binmore 1987). In a similar vein, Carlsson (2003) shows that if players' demands are made with a small random error term, then as the size of this error goes to zero the Nash bargaining solution is obtained as the limit of equilibrium strategies. Anbarci (2001) modifies the bargaining game so the dollar is still distributed if total demand exceeds 1 , but players receive less than their demands, and the 50-50 split is obtained. Another approach makes the bargaining game dynamic. Binmore et al. (1986) provide an alternating-offers model in which the Nash bargaining solution is the equilibrium outcome. Within a random matching model, Santamaria-Garcia (2004) and Robles (2008) take an evolutionary approach, relating the stable outcome to the Nash bargaining solution.

5 A player might choose to back down if announced demands are incompatible. Nash's game essentially assumes such costs are sufficiently large that neither player ever chooses to back down.
} 
As already noted, the key finding is that players' sets of demands must be balanced. Section 4 returns to Nash's original game and shows that if one player gains nothing from the bargaining, then even the finding that equilibrium demands are balanced need not hold.

\section{The Nash Demand Game}

The Nash Demand Game involving players 1 and 2 is described formally as follows. Player $i$ receives (von Neumann-Morgenstern) payoff $v_{i}\left(s_{1}, s_{2}\right)$ when player 1 demands $s_{1}$ and player 2 demands $s_{2}$. For any $\left(s_{1}, s_{2}\right) \in \mathbb{R}_{+} \times \mathbb{R}_{+}$, player $i$ 's realized payoff $u_{i}$ is

$$
u_{i}\left(s_{1}, s_{2}\right)= \begin{cases}v_{i}\left(s_{1}, s_{2}\right) & \text { if } s_{1}+s_{2} \leq 1 \\ 0 & \text { otherwise }\end{cases}
$$

The interpretation is that there exists a fixed sum of money, normalized to 1 , that players may be split between them if they can reach an agreement. Here $s_{1}$ and $s_{2}$ represent players' demands, and the function $u_{i}$ captures the rule that players get nonzero payoffs only if demands are compatible in the sense that $s_{1}+s_{2} \leq 1$. Reservation payoffs in the case of no agreement are normalized to 0 . Note that all demands $s>1$ are weakly dominated by $s=1$, and all demands $s \in(0,1]$ are undominated. Therefore, without loss of interest, henceforth, players' demands are restricted to the set $S \equiv[0,1]$.

The functions $v_{i}$ can be interpreted as reflecting both how the dollar is divided, given $\left(s_{1}, s_{2}\right)$, and player $i$ 's resulting value for such a split. For example, we might have, if players are risk neutral,

$$
\begin{gathered}
v_{i}\left(s_{1}, s_{2}\right)=s_{i}, \\
v_{i}\left(s_{1}, s_{2}\right)=s_{i}+\frac{1}{2}\left(1-s_{1}-s_{2}\right), \\
v_{i}\left(s_{1}, s_{2}\right)= \begin{cases}\frac{1}{2} & \text { if } s_{1}=0 \text { and } s_{2}=0 \\
\frac{s_{i}}{s_{1}+s_{2}} & \text { otherwise; }\end{cases}
\end{gathered}
$$

and, if players are risk-averse,

$$
v_{i}\left(s_{1}, s_{2}\right)=w_{i}\left(\tilde{v}_{i}\left(s_{1}, s_{2}\right)\right)
$$

where $w_{i}: \mathbb{R}_{+} \rightarrow \mathbb{R}_{+}$is a continuous strictly increasing concave function and $\tilde{v}_{i}$ is one of the functions of $v_{i}$ in (2)-(4). Specification (2) is Nash (1953)-if demands are compatible, players receive what they demand, with the possibility some money is not distributed. Specification (3) is from Roth (1985) — if demands are compatible, then each player receives his demand plus half of the unclaimed money. Specification (4) fully distributes the dollar, with players receiving shares proportional to their demands. 
Each player's set of mixed strategies, $\Sigma$, is the set of probability measures on $(S, \mathcal{A})$, where $\mathcal{A}$ is the Borel sigma-algebra on $S$. If a strategy $\sigma \in \Sigma$ assigns probability 1 to a single element $s \in S$, then it is a pure strategy and I may write $s$ instead of $\sigma$.

Definition 1 For any $\sigma \in \Sigma$, the support of $\sigma$ is defined as $\operatorname{supp}(\sigma) \equiv\{s \in S \mid$ $\forall \varepsilon>0, \sigma((s-\varepsilon, s+\varepsilon) \cap S)>0\} ; \sigma$ completely mixes over the set $A \subset S$ if $A=\operatorname{supp}(\sigma)$.

If the support $A$ of a strategy is a finite set, then that strategy assigns positive probability to every element of $A$. More generally, it can be shown that if $\sigma$ completely mixes over $A \subset S$, then $A$ is a compact set. I refer to the support of a mixed strategy as the set of demands possible under that strategy. ${ }^{6}$ Allowing for mixed strategies, define players' expected payoffs $U_{i}: \Sigma \times \Sigma \rightarrow \mathbb{R}$ by

$$
U_{i}\left(\sigma_{1}, \sigma_{2}\right)=\int_{S \times S} u_{i}\left(s_{1}, s_{2}\right) \mathrm{d} \sigma_{1}\left(s_{1}\right) \mathrm{d} \sigma_{2}\left(s_{2}\right), \quad i=1,2 .
$$

Definition $2\left(\sigma_{1}^{*}, \sigma_{2}^{*}\right) \in \Sigma \times \Sigma$ is an equilibrium if $U_{1}\left(\sigma_{1}^{*}, \sigma_{2}^{*}\right) \geq U_{1}\left(s_{1}, \sigma_{2}^{*}\right)$ and $U_{2}\left(\sigma_{1}^{*}, \sigma_{2}^{*}\right) \geq U_{2}\left(\sigma_{1}^{*}, s_{2}\right) \forall s_{1}, s_{2} \in S$.

This definition of equilibrium only requires no player can improve his payoff by using any particular pure strategy. Because the $U_{i}$ are linear in the probabilities, this is equivalent to requiring no player has another mixed strategy that increases his payoff [see Fudenberg and Tirole (1991, p. 11)].

N.B. Throughout I assume $v_{1}$ and $v_{2}$ satisfy Assumption 1.

Assumption 1 The functions $v_{1}$ and $v_{2}$ have the following properties:

(i) $v_{i}: S \times S \rightarrow \mathbb{R}_{+}$is Borel measurable.

(ii) $v_{1}(s, 0)>v_{1}(0,0)$ and $v_{2}(0, s)>v_{2}(0,0)$ for all $s>0$.

(iii) $v_{1}\left(s_{1}, s_{2}\right)$ is nonincreasing in $s_{2}$, nondecreasing in $s_{1}$ if $s_{2}=0$, and strictly increasing in $s_{1}$ if $s_{2}>0 ; v_{2}\left(s_{1}, s_{2}\right)$ is nonincreasing in $s_{1}$, nondecreasing in $s_{2}$ if $s_{1}=0$, and strictly increasing in $s_{2}$ if $s_{1}>0$.

Assumption 1(i) is a technical assumption ensuring expected payoffs are welldefined. Assumption 1(ii) implies both players announcing a demand of zero is not a pure-strategy equilibrium. Assumption 1(iii) implies players' interests are opposedif a pair of demands sums to less than one, then a player can gain by increasing his demand and this does not help (and possibly hurts) his rival. Functions (2)-(5) satisfy Assumption 1.

\footnotetext{
6 While this use of the word "possible" is correct when $A$ is a finite set, it can be imprecise when $A$ is infinite. For example, consider the set of demands $A \equiv\{1 / n \mid n=1,2,3, \ldots\} \cup\{0\}$ together with a strategy such that $\sigma(1 / n)>0$ for all $n$ and $\sum_{n} \sigma(1 / n)=1$. Then demand 0 is in the support of $\sigma$ but it is not in any sense "possible" under strategy $\sigma$. As a second example, consider a probability measure that assigns strictly positive probability to each rational number in $[0,1]$ and for which the total mass of the rationals in this interval is 1 . Then no irrational number is a possible demand under this strategy even though the support of the strategy is $[0,1]$. Having noted this imprecision, I will continue to use "possible demands" as shorthand for "the support of a player's distribution of demands."
} 


\section{Equilibria in the Nash Demand Game}

The equilibria defined above can be termed mixed-strategy equilibria (MSE) as each player's strategy is an element of $\Sigma$, the set of mixed strategies. If $\left(\sigma_{1}^{*}, \sigma_{2}^{*}\right)$ is an equilibrium and the supports of $\sigma_{1}^{*}$ and $\sigma_{2}^{*}$ are singletons, then $\left(\sigma_{1}^{*}, \sigma_{2}^{*}\right)$ can further be identified as a pure-strategy equilibrium (PSE). The set of PSE to this game is $\left\{\left(s_{1}, s_{2}\right) \in S \times S \mid s_{1}+s_{2}=1\right.$ or $\left.s_{1}=s_{2}=1\right\}$. Any equilibrium that is not a PSE I will refer to as a proper MSE. The strategy pair $\left(s_{1}, s_{2}\right)=(1,1)$ is the only PSE that yields disagreement. Arguably, such demands are unrealistic for either player as they ensure the other player a payoff of zero-and one might expect a player not to enter into the game if he expects no gain. In contrast, proper mixed strategy equilibria are significant in that they offer a possible explanation for disagreement while relying on demands that are more plausible as they offer potential benefits to both players.

The characterizations of equilibria in this section do not distinguish between PSE and proper MSE. The PSE were already noted above, so the value of these characterizations is that they also apply to proper MSE. I begin with an example in which both players completely mix over two distinct demands.

Example 1 Suppose $v_{i}\left(s_{1}, s_{2}\right)=s_{i}, i=1,2$. Let $a \in(0,1 / 2)$ be given and define $A \equiv\{a, 1-a\}$. The unique equilibrium in which each player completely mixes over $A$ has each player use strategy $\sigma^{*}$, where ${ }^{7}$

$$
\sigma^{*}(a)=\frac{a}{1-a} \text { and } \sigma^{*}(1-a)=\frac{1-2 a}{1-a} .
$$

For example, if $a=1 / 3$ then the corresponding MSE has each player demand either $1 / 3$ or $2 / 3$, each with probability $1 / 2$. The equilibrium can be understood as follows. If player 2 uses mixed strategy $\sigma^{*}$, then for each of player 1's possible demands, $s_{1}$, player 1's expected payoff is

$$
U_{1}\left(s_{1}, \sigma^{*}\right)= \begin{cases}s_{1} & \text { if } 0 \leq s_{1} \leq a \\ \frac{a}{1-a} s_{1} & \text { if } a<s_{1} \leq 1-a \\ 0 & \text { if } 1-a<s_{1} \leq 1 .\end{cases}
$$

Over the interval [0,a], $U_{1}\left(s_{1}, \sigma^{*}\right)$ is strictly increasing in $s_{1}$ and attains its maximum value of $a$ at $s_{1}=a$. Similarly, over the interval $(a, 1-a], U_{1}\left(s_{1}, \sigma^{*}\right)$ attains its maximum value of $a$ at $s_{1}=1-a$. And over the interval $(1-a, 1], U_{1}\left(s_{1}, \sigma^{*}\right)$ equals zero, which is strictly less than $a$. Thus, both $s_{1}=a$ and $s_{1}=1-a$ are player 1's best responses to player 2's mixed strategy, so player 1 is willing to randomize over these two demands. Similarly, player 2 is willing to randomize over $A$ if player 1 uses strategy $\sigma^{*}$. Thus, $\left(\sigma^{*}, \sigma^{*}\right)$ is an equilibrium in which each player completely mixes over $A$. To establish uniqueness, let $\left(\hat{\sigma}_{1}, \hat{\sigma}_{2}\right)$ be any equilibrium in which each player completely mixes over $A(=\{a, 1-a\})$. Player 1 's expected payoff is $U_{1}\left(a, \hat{\sigma}_{2}\right)=a$ when demanding $a$ and $U_{1}\left(1-a, \hat{\sigma}_{2}\right)=(1-a) \hat{\sigma}_{2}(a)$ when demanding $1-a$. To

\footnotetext{
7 I harmlessly abuse notation by writing $\sigma^{*}(a)$ rather than $\sigma^{*}(\{a\})$. Such abuse continues where convenient.
} 


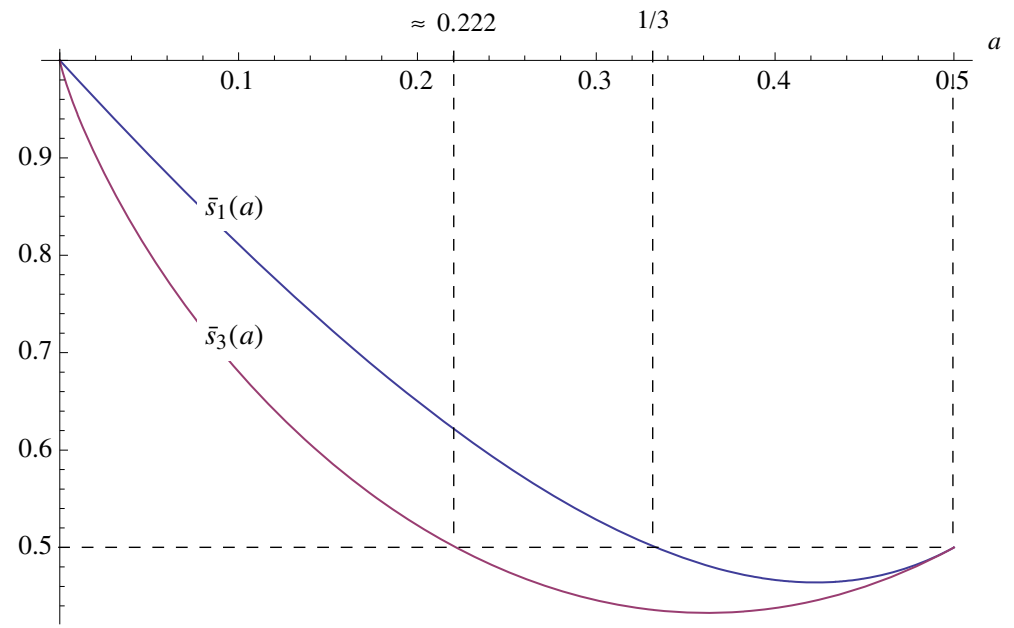

Fig. 1 Each player's expected demand in Examples 1 and 3

ensure player 1 is willing to randomize over these two possible demands, these payoffs must be equal, that is, $a=U_{1}\left(a, \hat{\sigma}_{2}\right)=U_{1}\left(1-a, \hat{\sigma}_{2}\right)=(1-a) \hat{\sigma}_{2}(a)$, which implies $\hat{\sigma}_{2}(a)=a /(1-a)$. Because $A$ has only two elements, $\hat{\sigma}_{2}$ assigns the complementary probability to $1-a$; that is, $\hat{\sigma}_{2}(1-a)=1-\hat{\sigma}_{2}(a)=(1-2 a) /(1-a)$. Thus, $\hat{\sigma}_{2}=\sigma^{*}$ as given in (7). Similarly, $\hat{\sigma}_{1}=\sigma^{*}$, thus establishing uniqueness.

For a given value of $a$, the MSE results in disagreement if and only if both players demand $1-a$, and this occurs with probability $(1-2 a)^{2} /(1-a)^{2}$, which decreases monotonically from 1 to 0 as $a$ increases from 0 to $1 / 2 .^{8}$ As $a$ increases from 0 toward $1 / 2$, the payoff to demanding $a$, namely $a$, is increasing. In this MSE, the payoff to demanding $1-a$ must also be increasing in $a$. But since $1-a$ decreases with $a$, this can happen only if the probability of agreement goes up, that is, the probability of disagreement falls as $a$ increases. Each player's expected demand equals

$$
\bar{s}_{1}(a)=a\left(\frac{a}{1-a}\right)+(1-a)\left(\frac{1-2 a}{1-a}\right)=\frac{1-3 a(1-a)}{1-a} .
$$

Figure 1 depicts the expected demand, $\bar{s}_{1}$, which is not monotonic in $a$. Between the two possibilities, $a$ and $1-a$, a player assigns greater probability to the lower number if and only if $1 / 3<a<1 / 2$.

The notable feature of Example 1 is that in equilibrium each possible demand by player 1 is balanced by a possible demand of player 2 that fully allocates the dollar. This feature does not require players' sets of possible demands to be identical, as assumed in Example 1.

\footnotetext{
8 As $a \rightarrow 0$, the MSE approaches the PSE in which each player announces $s=1$, which results in disagreement with probability one. As $a \rightarrow 1 / 2$, the MSE approaches the PSE in which each player announces $s=1 / 2$, which results in agreement with probability one.
} 
Definition 3 The sets $A$ and $B$ are balanced if the following is satisfied: $s \in A \Longleftrightarrow$ $1-s \in B$.

Derivation of MSE will generally be difficult, with equilibrium strategies depending on the particular choices of functions $v_{1}$ and $v_{2}$. As an alternative, I ask what sets of possible demands can arise in a MSE. Knowing which sets of demands are compatible with equilibrium is an essential first step in solving for equilibrium strategies. The critical property of players' sets of possible demands is that they be balanced, regardless of the functions $v_{1}$ and $v_{2}$ considered, as long as they satisfy Assumption 1 .

Proposition 1 Let $A$ and $B$ be nonempty closed subsets of $[0,1]$ such that $1 \notin A \cup B$. If $\left(\sigma_{1}^{*}, \sigma_{2}^{*}\right)$ is an equilibrium in which $\sigma_{1}^{*}$ completely mixes over $A$ and $\sigma_{2}^{*}$ completely mixes over $B$, then $A$ and $B$ are balanced.

The proof of Proposition 1 and proofs of subsequent propositions are in the Appendix. Proposition 1 is proven by showing that if $a \in A$ but $1-a \notin B$, then rather than ever demanding $a$, player 1 could demand $a+\varepsilon$, for some small $\varepsilon>0$, without increasing the chance of disagreement. Such an increase would raise player 1's payoff. Therefore, if in equilibrium player 1 fully mixes over $A$, then it must be that $1-a \in B$. The condition $1 \notin A \cup B$ in Proposition 1 is needed to exclude the PSE in which each player demands 1 with probability one-the sets $A=B=\{1\}$ are not balanced.

Players using balanced sets of demands can also be interpreted as independent attempts to choose among a given set of PSE. Suppose $A$ and $B$ are balanced sets of demands for players 1 and 2, respectively. The set $C \equiv\{(s, 1-s) \mid s \in A\}$ is simply the set of PSE in which player 1's strategy belongs to $A$. Because $A$ and $B$ are balanced, $B$ coincides with the set of strategies player 2 uses in the PSE in $C$. Thus, an equilibrium in which player 1 completely mixes over $A$ and player 2 completely mixes over $B$ may interpreted as players realizing an equilibrium in $C$ should be implemented, but coordination failure leaves them independently randomizing over their sets of pure strategies associated with $C$. This is the natural extension of players' failure to coordinate on one of two equilibria in Roth's experiments described above (see also Example 2 below).

The following proposition provides the converse to Proposition 1 for the case in which sets $A$ and $B$ are finite. This result is key to proving the converse of Proposition 1.

Proposition 2 Let $A$ and $B$ be nonempty finite subsets of $[0,1]$ such that $1 \notin A \cup B$. If the sets $A$ and $B$ are balanced, then there exists a unique equilibrium in which player 1 completely mixes over A and player 2 completely mixes over $B$.

The condition $1 \notin A \cup B$ in Proposition 2 is needed to exclude the case in which $A=B=\{0,1\}$. These sets are balanced, but if $v_{1}(0,1)=v_{2}(1,0)=0$ (as in (2)), then there is no MSE in which both players completely mix over $\{0,1\} .{ }^{9}$ Propositions 1 and 2 yield the following corollary.

\footnotetext{
9 However, if $v_{1}(0,1)>0$ and $v_{2}(1,0)>0$, then there is indeed an equilibrium in which each player completely mixes over $\{0,1\}$. This issue is discussed further in Sect. 4 .
} 
Fig. 2 The matrix game associated with Example 2

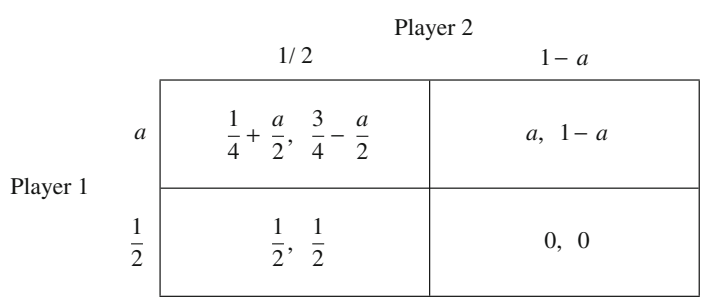

Corollary 1 Let $A$ and $B$ be nonempty finite subsets of $[0,1]$ such that $1 \notin A \cup B$. There exists an equilibrium in which player 1 completely mixes over $A$ and player 2 completely mixes over $B$ if and only if the sets $A$ and $B$ are balanced. If such an equilibrium exists, it is unique.

The intuition for uniqueness of equilibrium in Corollary 1 can be understood by considering $A=\left\{a_{j}\right\}_{j=1}^{n}$, where $0<a_{1}<a_{2}<\cdots<a_{n-1}<a_{n}<1$. For demands to be balanced, player 2's set of demands is $B=\left\{1-a_{n}, \ldots, 1-a_{1}\right\}$. Let $\bar{u}_{2}>0$ denote player 2's payoff at equilibrium $\left(\sigma_{1}^{*}, \sigma_{2}^{*}\right)$ that completely mixes over $A$ and $B$. Now suppose player 2 demands $1-a_{1}$; the only chance for agreement is if player 1 demands $a_{1}$, and player 2's willingness to make this demand requires $\bar{u}_{2}=U_{2}\left(\sigma_{1}^{*}, 1-a_{1}\right)=v_{2}\left(a_{1}, 1-a_{1}\right) \sigma_{1}^{*}\left(a_{1}\right)$, which determines $\sigma_{1}^{*}\left(a_{1}\right)$. If player 2 makes the second-highest demand in $B$, namely $1-a_{2}$, the only possibilities for agreement are if player 1 demands either $a_{1}$ or $a_{2}$. We have already determined $\sigma_{1}^{*}\left(a_{1}\right)$, so the condition

$$
\bar{u}_{2}=U_{2}\left(\sigma_{1}^{*}, 1-a_{2}\right)=v_{2}\left(a_{1}, 1-a_{2}\right) \sigma_{1}^{*}\left(a_{1}\right)+v_{2}\left(a_{2}, 1-a_{2}\right) \sigma_{1}^{*}\left(a_{2}\right)
$$

uniquely determines $\sigma_{1}^{*}\left(a_{2}\right)$. Descending in this manner through the set of player 2's demands uniquely determines $\sigma_{1}^{*}$. Similarly, $\sigma_{2}^{*}$ is uniquely determined.

In the following example, players randomize over balanced but nonidentical sets of demands.

Example 2 (Roth 1985) Suppose $v_{1}$ and $v_{2}$ are given by (3). Let $a \in(0,1 / 2)$ be given and define $A \equiv\{a, 1 / 2\}$ and $B \equiv\{1 / 2,1-a\}$. The sets $A$ and $B$ are balanced. The associated $2 \times 2$ matrix game is shown in Fig. 2, and the unique completely mixed equilibrium is readily found to be $\left(\sigma_{1}^{*}, \sigma_{2}^{*}\right)$, where

$$
\sigma_{1}^{*}(a)=\frac{2}{3-2 a} \quad \sigma_{1}^{*}(1 / 2)=\frac{1-2 a}{3-2 a}
$$

and

$$
\sigma_{2}^{*}(1 / 2)=\frac{4 a}{1+2 a} \quad \sigma_{2}^{*}(1-a)=\frac{1-2 a}{1+2 a} .
$$

Proposition 2 implies $\left(\sigma_{1}^{*}, \sigma_{2}^{*}\right)$ is the unique MSE in which player 1 completely mixes over $A$ and player 2 completely mixes over $B$. It is interesting to note that in 


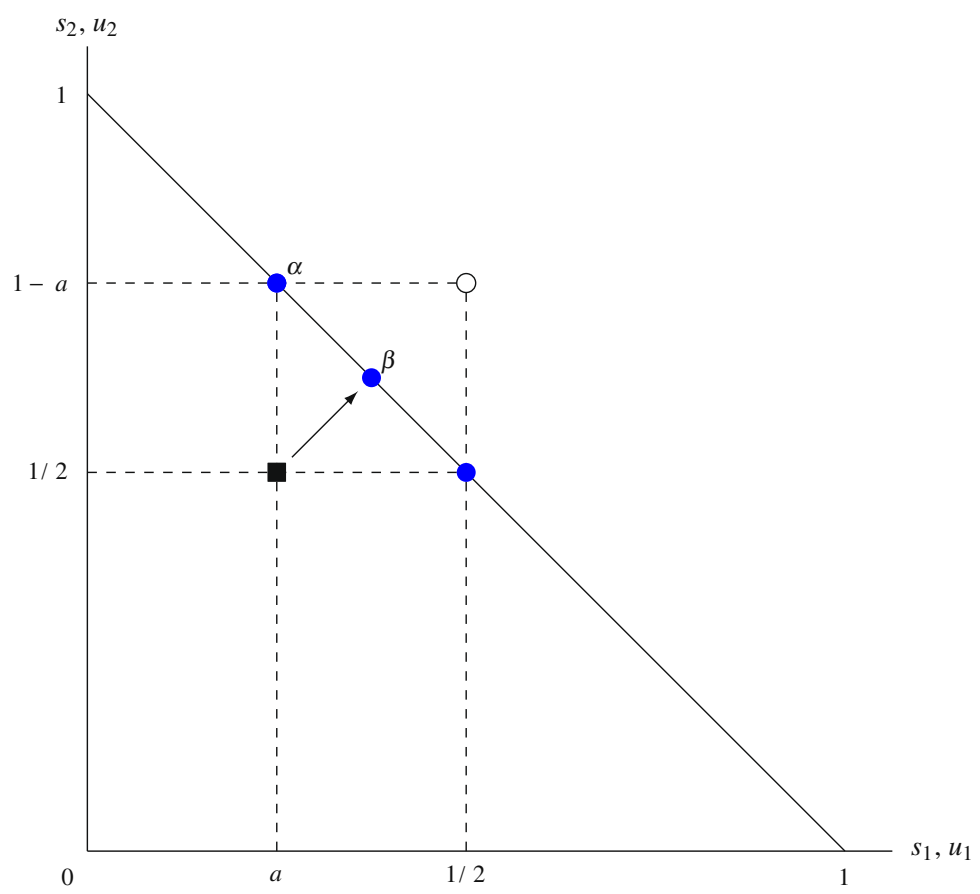

Fig. 3 Demands and payoffs in the Roth example

contrast to Examples 1 and 3 (below), in Example 2 the probability of disagreement, $(1-2 a)^{2} /\left(3+4 a-4 a^{2}\right)$, does not exceed $1 / 3$. That the probability of disagreement is bounded away from 1 can be understood as follows.

Figure 3 depicts demand and payoff combinations Roth's example. The possible combinations of demands are represented by the intersections of the four dashed lines. The strictly positive payoff possibilities are represented by the shaded circles (here the demand pair $(a, 1 / 2)$ is marked by the black box and the corresponding pair of payoffs is the shaded circle lying to the northeast). The only demand combination for which players' payoffs are zero is shown by the unshaded circle. Notice that player 2 , by demanding $1 / 2$ can assure agreement and his expected payoff will be at least $1 / 2$. This is true for all $a \in(0,1 / 2)$. Now, as $a$ decreases toward 0 , payoff combinations labeled $\alpha$ and $\beta$ move northwest and player 2's expected payoff is bounded away from 0 (in fact, it is sure to exceed $1 / 2$ ), and the realized payoff is uniformly bounded above. Therefore, the probability of disagreement must be bounded away from 1, even as $a$ decreases toward 0 .

Depicting Example 1 in a similar diagram, one quickly sees why the probability of disagreement ranges over the full interval $[0,1]$. The analogous argument also applies in Example 3 below where players completely mix over the interval $[a, 1-a]$ and the probability of disagreement varies from 0 to 1 . Furthermore, if instead, say, players completely mix over the intervals $A=[a, 1 / 2]$ and $B=[1 / 2,1-a]$, where $a \in(0,1 / 2)$, it readily follows that the probability of disagreement is bounded away 
from $1 .{ }^{10}$ This reasoning does not depend on the set of conceivable payoff combinations having a linear frontier or even being convex. To keep the probability of disagreement bounded away from 1, the key is that one player's set of possible payoffs remains bounded away from 0 . This point is discussed further following Example 5.

To extend Proposition 2 to general closed sets $A$ and $B$, I approximate $A$ and $B$ by finite balanced subsets. For these finite approximations, Proposition 2 ensures existence of a unique equilibrium with strategies completely mixed over the balanced sets. The general case is treated as the limit of finite approximations. To extend results from the finite case, I must assume the payoffs are sufficiently continuous. The following suffices.

Assumption 2 The functions $v_{1}$ and $v_{2}$ are continuous on $(0,1] \times(0,1]$.

Functions (2)-(4) satisfy Assumption 2.

Proposition 3 Suppose Assumption 2 is satisfied. Let $A$ and $B$ be nonempty closed subsets of $[0,1]$ such that $1 \notin A \cup B$. If the sets $A$ and $B$ are balanced, then there exists an equilibrium $\left(\sigma_{1}^{*}, \sigma_{2}^{*}\right)$ in which $\sigma_{1}^{*}$ completely mixes over $A$ and $\sigma_{2}^{*}$ completely mixes over $B$.

Propositions 1 and 3 yield the following corollary.

Corollary 2 Suppose Assumption 2 is satisfied. Let $A$ and $B$ be nonempty closed subsets of $[0,1]$ such that $1 \notin A \cup B$. There exists an equilibrium in which player 1 completely mixes over $A$ and player 2 completely mixes over $B$ if and only if the sets $A$ and $B$ are balanced.

The equilibria of Corollary 2 are quasi-strong (Harsanyi 1973); that is, the support of each player's equilibrium strategy contains all of the pure-strategy best responses to the rival's equilibrium strategy.

Inefficiency of equilibria is common, and it arises here when there is a positive probability of disagreement. Suppose the functions $v_{1}$ and $v_{2}$ satisfy the following condition:

$\forall a, b \in[0,1]$ and $\forall \varepsilon>0, \exists \delta<\varepsilon / 2$ s.t. $v_{i}(a+\delta, b+\delta)>v_{i}(a, b), \quad i=1,2$.

This condition simply requires that a player value his own (incremental) gain more strongly than the other's. Consider any equilibrium $\left(\sigma_{1}^{*}, \sigma_{2}^{*}\right)$ of Corollary 2 , and let $\underline{a}=\min \{a \mid a \in A\}, \bar{a}=\max \{a \mid a \in A\}$ and $\underline{b}=\min \{b \mid b \in B\}$, and $\bar{u}_{i} \equiv$ $U_{i}\left(\sigma_{1}^{*}, \sigma_{2}^{*}\right), i=1,2$. Because $\bar{u}_{1}>0$, it must be that $\underline{b}$ is an atom of $\sigma_{2}^{*}$; similarly, $\underline{a}$ must be an atom of $\sigma_{1}^{*}$. Consequently,

$$
\bar{u}_{1}=U_{1}\left(\underline{a}, \sigma_{2}^{*}\right)=\mathrm{E}\left[v_{1}\left(\underline{a}, s_{2}\right) \mid \sigma_{2}^{*}\right] \leq v_{1}(\underline{a}, \underline{b}),
$$

\footnotetext{
10 The equilibrium in this continuous version of Roth's example is derived in the Appendix. The probability of disagreement is shown to equal $1-(\sqrt{a}+\sqrt{1-a}) / \sqrt{2}$.
} 
where the second equality follows because agreement is sure to occur, and the inequality follows because $v_{1}$ is nonincreasing in $b$; similarly, $\bar{u}_{2} \leq v_{2}(\underline{a}, \underline{b})$. If $A$ contains at least two elements, then we also have $1-(\underline{a}+\underline{b})=1-(\underline{a}+1-\bar{a})=(\bar{a}-\underline{a})>0$, where the first equality follows by balancedness. By (8) it now follows that there is $\delta \in(0,(\bar{a}-\underline{a}) / 2))$ such that if players announce $(\underline{a}+\delta, \underline{b}+\delta)$, then demands will be satisfied and both players will enjoy payoffs greater than at $\left(\sigma_{1}^{*}, \sigma_{2}^{*}\right)$. Thus, $\left(\sigma_{1}^{*}, \sigma_{2}^{*}\right)$ is inefficient.

With Corollary 2, we see there is now a simple two-step procedure to constructing equilibria where both players benefit. First, players' balanced sets of demands are chosen-and for this step utilities are not relevant. Second, equilibrium probabilities over these sets of demands are determined-and here the particular utility functions are used. When players' payoff functions $v_{i}$ depend on only their own demands, this characterization is straightforward, as shown next in Proposition 4. For this characterization it is convenient to introduce the cumulative distribution function associated with a mixed strategy. For any $\sigma \in \Sigma$, the associated cumulative distribution function (cdf), $F$, is defined by $F(s) \equiv \sigma((-\infty, s])$, for all real numbers $s$; note that $F(s)=0$ for all $s<0$ and $F(s)=1$ for all $s \geq 1$.

Proposition 4 Suppose $v_{i}\left(s_{1}, s_{2}\right)=w_{i}\left(s_{i}\right)$, where $w_{i}: \mathbb{R}_{+} \rightarrow \mathbb{R}_{+}$is continuous and strictly increasing on $\mathbb{R}_{++}, i=1,2$. Let $A$ and $B$ be balanced nonempty closed subsets of $[0,1]$ such that $1 \notin A \cup B$. Define $\underline{a} \equiv \min \{a \mid a \in A\}, \bar{a} \equiv \max \{a \mid a \in A\}$, $\underline{b} \equiv \min \{b \mid b \in B\}$, and $\bar{b} \equiv \max \{\bar{b} \mid b \in B\}$. Then the unique equilibrium $\left(\sigma_{1}^{*}, \sigma_{2}^{*}\right)$ in which $\sigma_{1}^{*}$ completely mixes over $A$ and $\sigma_{2}^{*}$ completely mixes over $B$ is described by the corresponding cdfs, $F_{1}$ and $F_{2}$, given as follows:

$$
F_{1}(s)= \begin{cases}0 & \text { if } s<\underline{a} \\ \frac{w_{2}(\underline{b})}{w_{2}(1-s)} & \text { if } s \in A \\ \underset{\substack{a \in A \\ \text { s.t. } a<s}}{\max } \frac{w_{2}(\underline{b})}{w_{2}(1-a)} & \text { if } s>\underline{a} \text { and } s \notin A\end{cases}
$$

and

$$
F_{2}(s)= \begin{cases}0 & \text { if } s<\underline{b} \\ \frac{w_{1}(\underline{a})}{w_{1}(1-s)} & \text { if } s \in B \\ \max _{\substack{b \in B \\ \text { s.t. } b<s} \frac{w_{1}(\underline{a})}{w_{1}(1-b)}} & \text { if } s>\underline{b} \text { and } s \notin B .\end{cases}
$$

Complementing Example 1, the next example has players completely mix over an interval of demands.

Example 3 Suppose $v_{i}\left(s_{1}, s_{2}\right)=s_{i}, i=1,2$. Let $a \in(0,1 / 2]$ be given and define $A \equiv[a, 1-a]$. Proposition 3 implies the unique equilibrium in which each player 
completely mixes over $A$ has each player announce a demand according to the cumulative distribution function $F$ given by

$$
F(s)= \begin{cases}0 & \text { if } s<a \\ \frac{a}{1-s} & \text { if } a \leq s \leq 1-a \\ 1 & \text { if } 1-a<s \leq 1\end{cases}
$$

which on the interval $(a, 1-a]$ has the corresponding density function $f(s)=$ $a(1-s)^{-2}$. In this MSE, a player demands $a$ with probability $a /(1-a)$ and announces a demand from $(a, 1-a$ ] according to density $f$. If player 1 , say, announces demand $a$ there is sure to be agreement. So, suppose player 1 announces demand $s \in(a, 1-a]$. There is disagreement if and only if player 2 demands more than $1-s$, which occurs with probability $1-F(1-s)$; because player 1's demand $s>a$ is distributed according to density $f$, in equilibrium players' demands disagree with probability

$$
\begin{aligned}
\operatorname{Pr}(\text { disagreement }) & =\int_{a}^{1-a}(1-F(1-s)) f(s) \mathrm{d} s \\
& =\int_{a}^{1-a}\left(1-\frac{a}{1-(1-s)}\right) \frac{a}{(1-s)^{2}} \mathrm{~d} s \\
& =\left[\frac{a(1-a)}{1-s}+\left.a^{2} \log \left(\frac{1-s}{s}\right)\right|_{a} ^{1-a}\right. \\
& =1-2 a-2 a^{2} \log \left(\frac{1-a}{a}\right),
\end{aligned}
$$

where $\log (x)$ denotes the natural $\operatorname{logarithm}$ of $x$. The probability of disagreement decreases monotonically from 1 to 0 as $a$ increases from 0 to $1 / 2$. Each player's expected demand equals

$$
\begin{aligned}
\bar{s}_{3}(a) & =a F(a)+\int_{a}^{1-a} s f(s) \mathrm{d} s \\
& =\frac{a^{2}}{1-a}+\left[\frac{a}{1-s}+\left.a \log (1-s)\right|_{a} ^{1-a}\right. \\
& =(1-a)+a \log \left(\frac{a}{1-a}\right) .
\end{aligned}
$$


The pattern of the expected demand, depicted in Fig. 1, is similar to that in Example 1 . Furthermore, for every $a \in(0,1 / 2)$, the probability of disagreement and the expected demand are strictly less in Example 3 than in Example 1. This occurs because, although the equilibrium strategies of Examples 1 and 3 assign the same probability to the demand $a$, Example 1 concentrates the remaining probability at $1-a$, while Example 3 distributes it continuously over the interval $(a, 1-a]$. The equilibrium strategies may be interpreted as follows. Each player demands $a$ for sure, and then with (independent) probability $\frac{1-2 a}{1-a}$ asks for a little more. In Example 1 this "something extra" is $1-2 a$, and disagreement occurs if both players ask for something extra. In Example 3, this "something extra" is drawn from the interval $(0,1-2 a]$; therefore, some extra demands can still be accommodated without exceeding the allotment of 1 , and so disagreement need not occur. Thus, the probability of disagreement is less in Example 3 than in Example 1. Similarly, because with probability 1 the extra amount demanded in Example 3 is less than that in Example 3, it follows that the expected demand is less in Example 3 than in Example 1. Similar patterns can be seen in the continuous version of Roth's example (see the Appendix).

This section concludes with an example that illustrates mixed-strategy equilibria that can arise in the provision of a discrete public good, even in the presence complete information.

Example 4 (Private provision of a public good) Suppose a public good will be provided if citizens' contributions fully fund it. There are two citizens, 1 and 2 . Suppose person $i$ 's strictly positive private value for the good is $u_{i}, i=1,2$. Assume the cost of the good is 1 , where $u_{1}+u_{2}>1$. Let $c_{i}$ denote player $i$ 's contribution. If contributions total at least 1 , the good is provided; if not, contributions are refunded. Suppose citizen $i$ 's payoff is $v_{i}=u_{i}-c_{i}$ if the good is provided and 0 otherwise. There are no sidepayments between players. The foregoing is common knowledge. This example allows players to make any nonnegative contributions; Palfrey and Rosenthal (1984) studied this game for the case in which players' contributions are either 0 or some fixed positive amount.

The equilibria of this game can be described by recasting it as a Nash demand game. The achievable total surplus is $U_{0} \equiv u_{1}+u_{2}-1>0$. Without sidepayments, players conceivably might demand payoffs $s_{i} \in\left[0, u_{i}\right]$; given demand $s_{i}$, player $i$ 's corresponding willingness to contribute is $c_{i}=u_{i}-s_{i}$. For simplicity, I restrict attention to equilibria in which both players earn strictly positive expected payoffs. A pair of utility demands, $\left(s_{1}, s_{2}\right)$, is balanced if $U_{0}=s_{1}+s_{2}$, which is equivalent to $c_{1}+c_{2}=1$. Now focus on contributions, and define

$$
C_{1} \equiv\left(1-u_{2}, u_{1}\right) \cap[0,1] \text { and } C_{2} \equiv\left(1-u_{1}, u_{2}\right) \cap[0,1]
$$

In equilibrium, player 1 would not contribute more than 1 ; and to earn a positive payoff he must contribute less than $u_{1}$. At the same time, if player 2 is to earn a positive payoff, player 1 must contribute more than $1-u_{2}$ (if player 1 contributes less, he knows the good will be underfunded and so his payoff will be zero). These considerations imply that if equilibrium payoffs are to be positive, then player 1's possible contributions will be in set $C_{1}$; analogous considerations apply to player 2 and $C_{2}$. 
By the assumptions in the first paragraph of this example, $C_{1}$ and $C_{2}$ are nonempty nondegenerate intervals. Moreover, these intervals are balanced. Let $A$ be a nonempty closed subset of $C_{1}$ and $B$ a nonempty closed subset of $C_{2}$. The earlier analysis shows that if the sets $A$ and $B$ are balanced, then there exists a unique equilibrium $\left(\sigma_{1}^{*}, \sigma_{2}^{*}\right)$ in which $\sigma_{1}^{*}$ completely mixes over $A$ and $\sigma_{2}^{*}$ completely mixes over $B$. If set $A$ has more than one element, the equilibrium will be inefficient as there is positive probability the good is not provided. Finally, just considering players' randomization over intervals, because $C_{1}$ is a nondegenerate interval, there are (uncountably) many nondegenerate closed intervals within $C_{1}$ so there are (uncountably) many nondegenerate mixed-strategy equilibria to this game.

\section{Mixed-strategy equilibria when one player gains nothing}

The preceding analysis characterized equilibria in which neither player's strategy had 1 in its support. This condition was sufficient for players' equilibrium payoffs to be strictly positive. This condition is not always needed-for example, in Example 2, we can allow $a=0$, so players completely mix over $A=\{0,1 / 2\}$ and $B=\{1 / 2,1\}$. This is possible because player 1 can obtain a positive payoff even when demanding 0 . Indeed, with some caveats, one could recast most of the analysis of the previous section as characterizing equilibria in which each player's payoff exceeds his payoff at the disagreement outcome. This section explores the possibilities for equilibria when one player is sure to get the disagreement payoff. The reader may find, as I do, these equilibria highly implausible — why would one player even venture into the game if he expects, with probability one, not to do better than the disagreement outcome? Nevertheless, for completeness this section investigates what can be said about the behavior of such a player. We saw in Sect. 3 that when both players gain from the bargaining, their sets of possible demands must be balanced. In contrast, when one player does not gain, balancedness of strategies no longer applies and equilibrium randomization over given sets of demands need not be unique.

For simplicity, throughout this section I assume the payoff functions are $v_{i}\left(s_{1}, s_{2}\right)=s_{i}$, as assumed by Nash (1953). We will see that if one player expects no gain, then the other must be demanding 1 with probability 1 . Furthermore, a series of examples will show that if one player demands 1 for sure, then for the other player any closed set of demands containing 0 can be supported in equilibrium as that player's set of possible demands. The next proposition shows that if 1 is in the support of either player's strategy, then at least one player demands 1 with probability one.

Proposition 5 Suppose $v_{i}\left(s_{1}, s_{2}\right)=s_{i}, i=1,2$. Suppose $\left(\sigma_{1}^{*}, \sigma_{2}^{*}\right)$ is an equilibrium in which $\sigma_{1}^{*}$ completely mixes over $A$ and $\sigma_{2}^{*}$ completely mixes over $B$.

1. If $1 \in A$ and $1 \notin B$, then $\sigma_{1}^{*}(1)=1$.

2. If $1 \notin A$ and $1 \in B$, then $\sigma_{2}^{*}(1)=1$.

3. If $1 \in A$ and $1 \in B$, then $\sigma_{1}^{*}(1)=1$ or $\sigma_{2}^{*}(1)=1$.

The logic behind the proof of Proposition 5 can be seen by considering part 1 for the case in which 1 is an atom of $\sigma_{1}^{*}$, i.e., $\sigma_{1}^{*}(1)>0$. Because $1 \notin B$ it follows that there is some demand that yields player 1 a positive payoff; hence, $U_{1}\left(\sigma_{1}^{*}, \sigma_{2}^{*}\right)>0$. 
Because $U_{1}\left(\sigma_{1}^{*}, \sigma_{2}^{*}\right)>0$ and 1 is an atom of $\sigma_{1}^{*}$, it now follows that 0 is an atom of $B$, implying $U_{2}\left(\sigma_{1}^{*}, \sigma_{2}^{*}\right)=0$. For there to be no demand yielding player 2 a positive payoff, it must be that $\sigma_{1}^{*}(1)=1$.

The remainder of this section shows when one player, say player 1, demands 1 , little more can be said about the equilibrium sets of demands. The following examples culminate in showing that player 1's certain demand of 1 can be "rationalized" by an equilibrium strategy for player 2 that completely mixes over any closed subset $B$ of $[0,1]$ that contains 0 . Moreover, there are infinitely many equilibrium strategies in which player 2 has this same set of possible demands.

I begin this progression of examples by showing that if one player demands 1 for sure, then balancedness of the supports and uniqueness of the equilibrium (with those supports) is no longer assured. The following example shows that there are infinitely many equilibria in which one player demands 1 for sure and the other completely mixes over $[0,1]$.

Example 5 Suppose $v_{i}\left(s_{1}, s_{2}\right)=s_{i}, i=1,2$. Define mixed strategies $\sigma_{1}^{*}$ and $\sigma_{2, t}^{*}$ as follows. For player $1, \sigma_{1}^{*}(1)=1$. For player 2 the cdf, $F_{t}$, associated with $\sigma_{2, t}^{*}$ is

$$
F_{t}(s)=\frac{t}{1-(1-t) s} \text { if } 0 \leq s \leq 1
$$

where $t \in(0,1)$ is a parameter that specifies the probability with which player 2 announces a demand of 0 . Because player 1 demands 1 for sure, player 2 earns a payoff of 0 regardless of his own demand. In particular, for player 2 the cdf $F_{t}$ is a best response to player 1's demand. To see player 1's strategy is the unique best response to $\sigma_{2, t}^{*}$, observe that for any $s<1$,

$$
U_{1}\left(1, \sigma_{2, t}^{*}\right)-U_{1}\left(s, \sigma_{2, t}^{*}\right)=t-s F_{t}(1-s)=\frac{(1-s) t^{2}}{s+(1-s) t}>0 .
$$

Here $\operatorname{supp}\left(\sigma_{1}^{*}\right)=\{1\}$ and $\operatorname{supp}\left(\sigma_{2, t}^{*}\right)=[0,1]$, so, while $\left(\sigma_{1}^{*}, \sigma_{2, t}^{*}\right)$ is an equilibrium, the players' sets of possible demands are not balanced. Moreover, $\left\{\left(\sigma_{1}^{*}, \sigma_{2, t}^{*}\right)\right\}_{t \in(0,1)}$ represents an infinite set of equilibria in which player 1 demands 1 for sure and player 2 completely mixes over $[0,1]$. The probability of disagreement is $1-t$.

When player 1 demands 1 for himself, the equilibrium probability of agreement can lie anywhere in the interval $[0,1]$. To see this, first observe that, because in Example 5 the parameter $t$ is the probability of agreement, the equilibrium probability of agreement can lie anywhere in $(0,1)$. Alternatively, if player 2 demands 0 for sure, then the probability of agreement is 1 ; if he demands 1 for sure, it is 0 .

The following example shows that there are also infinitely many equilibria in which one player demands 1 for sure and the other player completely mixes over the interval $[0,1-p]$, where $p \in(0,1)$ is a parameter.

Example 6 Suppose $v_{i}\left(s_{1}, s_{2}\right)=s_{i}, i=1,2$. Fix $p \in(0,1)$ and define mixed strategies $\sigma_{1}^{*}$ and $\sigma_{2, t}^{*}$ as follows. For player $1, \sigma_{1}^{*}(1)=1$. For player 2 the cdf, $F_{t}$, associated 
with $\sigma_{2, t}^{*}$ is given as follows:

$$
F_{t}(s)= \begin{cases}p+(1-p)\left(\frac{p s}{(1-p)(1-s)}\right)^{t} & \text { if } 0 \leq s \leq 1-p \\ 1 & \text { if } 1-p<s \leq 1\end{cases}
$$

where $t>1$ is a parameter. Player 2's strategy is obviously a best response to player 1's. To see player 1's strategy is a best response to $\sigma_{2, t}^{*}$, first observe all demands $s \leq p$ are sure to be satisfied, so among these player 1's best choice is $s=p$, with $U_{1}\left(p, F_{t}\right)=p$. A demand $s=1$ is equally as good as $s=p: U_{1}\left(1, F_{t}\right)=1 \times F_{t}(0)=p=U_{1}\left(p, F_{t}\right)$. Next observe that for any $p<s<1$,

$$
\begin{aligned}
U_{1}\left(s, \sigma_{2, t}^{*}\right) & =s F_{t}(1-s) \\
& =s p+s(1-p)\left(\frac{p(1-s)}{(1-p) s}\right)^{t} \\
& <s p+s(1-p)\left(\frac{p(1-s)}{(1-p) s}\right) \\
& =p
\end{aligned}
$$

where (11) follows because $0<\frac{p(1-s)}{(1-p) s}<1$ and $t>1$. Thus, player 1's best responses to $F_{t}$ are $s=1$ and $s=p$. Consequently, $\left(\sigma_{1}^{*}, \sigma_{2, t}^{*}\right)$ is an equilibrium, with $\operatorname{supp}\left(\sigma_{1}^{*}\right)=$ $\{1\}$ and $\operatorname{supp}\left(\sigma_{2, t}^{*}\right)=[0,1-p]$. These supports are not balanced, and because there is such an equilibrium for every $t \in(0,1)$, there are infinitely many equilibria with these supports. Note that player 2's strategy places mass $p$ on demand 0 , so $p$ is also the probability of agreement.

Building on the two previous examples, the final example shows for any closed set $B \subset[0,1]$ with $0 \in B$, there is an equilibrium in which one player demands 1 for sure and the other completely mixes over $B$.

Example 7 Suppose $v_{i}\left(s_{1}, s_{2}\right)=s_{i}, i=1,2$. Let $A=\{1\}$ and let $B$ be any nonempty closed subset of $[0,1]$ with $0 \in B$. Then there exists a MSE in which player $1 \mathrm{com}-$ pletely mixes over $A$ and player 2 completely mixes over $B$. Moreover, if $B$ contains at least two points, then there exist infinitely many such equilibria.

This conclusion can be understood as follows. If $B$ contains only one point it must be 0 , so the PSE in which player 1 demands 1 and player 2 demands 0 is the unique equilibrium. If instead $B$ contains at least 2 points, let $p$ be such that $1-p$ is the greatest element of $B$. It follows from Examples 5 and 6 that there are infinitely many equilibria in which player 1 demands 1 for sure and player 2 completely mixes over the interval $[0,1-p]$. Let $F$ be such a strategy for player 2 . Define the $\operatorname{cdf} G$ as follows:

$$
G(s)=\max _{\substack{b \in B \\ \text { s.t. } b \leq s}} F(b)
$$




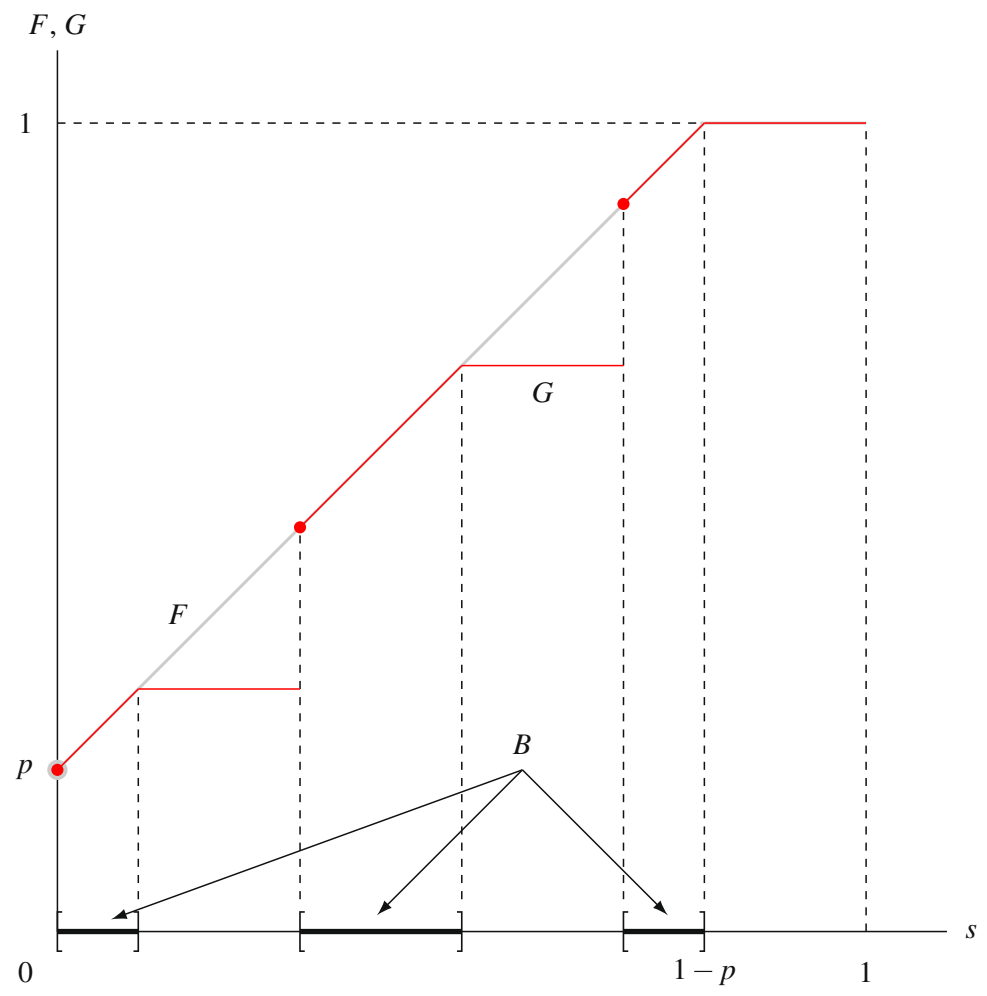

Fig. 4 Constructing $G$ from $F$

Figure 4 shows the relationship between cdfs $F$ and $G$. Then $\operatorname{supp}(G)=B$, and because $G(0)=F(0)$ but otherwise shifts probability to higher values (in the sense of firstorder stochastic dominance), it follows that 1 and $p$ are player 1's best responses to $G$. Thus, it is an equilibrium for player 1 to demand 1 and player 2 to announce a demand according to $G$. Because this procedure is applicable to each of the infinitely many equilibrium cdfs completely mixed over $[0,1-p]$, it follows that there are infinitely many equilibria in which player 1 announces 1 and player 2 completely mixes over $B$. Note that only for the case of $B=[0,1]$ is the equilibrium quasi-strong.

\section{Conclusion}

The literature on bargaining has focused on pure-strategy equilibria. Of these, the Nash bargaining solution commands center stage. Nevertheless, in the Nash Demand Game, there exists an abundance of proper mixed-strategy equilibria, which allow for equilibrium outcomes of agreement or disagreement. This paper has shown such equilibria can be constructed in two steps. First, players' sets of possible demands are chosen to be balanced, and if we exclude 1 from these sets then payoffs are sure to be larger in equilibrium than the disagreement payoff. Second, each player's probability 
distribution over his set of possible demands is determined by the condition that the other player be willing to play each of his possible demands. It was shown that almost any set of balanced demands can support a mixed-strategy equilibrium, yielding any probability of disagreement between 0 and 1 .

Open Access This article is distributed under the terms of the Creative Commons Attribution Noncommercial License which permits any noncommercial use, distribution, and reproduction in any medium, provided the original author(s) and source are credited.

\section{Appendix}

Proof of Proposition 1. Suppose $\left(\sigma_{1}^{*}, \sigma_{2}^{*}\right)$ is an equilibrium in which $\sigma_{1}^{*}$ completely mixes over $A$ and $\sigma_{2}^{*}$ completely mixes over $B$. Assumption 1 and $1 \notin A \cup B$ imply $\sigma_{i}^{*}(0)<1, i=1,2$. I now show $s \in A \Longrightarrow 1-s \in B$. The symmetric condition " $s \in B \Longrightarrow 1-s \in A$ " is proven analogously.

Suppose the proposition is false; in particular, suppose there exists $a^{\prime} \in A$ such that $1-a^{\prime} \notin B$. Because $B$ is closed and $0<a^{\prime}<1$, there exists $\varepsilon>0$ such that $0<a^{\prime}-2 \varepsilon, a^{\prime}+2 \varepsilon<1$, and $\left(1-a^{\prime}-2 \varepsilon, 1-a^{\prime}+2 \varepsilon\right) \cap B=\varnothing$, implying $\sigma_{2}^{*}\left(\left[1-a^{\prime}-\varepsilon, 1-a^{\prime}+\varepsilon\right]\right)=0$. Consider any $s^{\prime \prime} \in\left(a^{\prime}-\varepsilon, a^{\prime}+\varepsilon\right)$ :

$$
\begin{aligned}
U_{1}\left(\sigma_{1}^{*}, \sigma_{2}^{*}\right) \geq & U_{1}\left(a^{\prime}+\varepsilon, \sigma_{2}^{*}\right) \quad(\text { definition of equilibrium) } \\
& =\int_{S} u_{1}\left(a^{\prime}+\varepsilon, s_{2}\right) \mathrm{d} \sigma_{2}^{*}\left(s_{2}\right) \quad\left(\text { definition of } U_{1}\right) \\
& =\int_{\left[0,1-a^{\prime}-\varepsilon\right]} v_{1}\left(a^{\prime}+\varepsilon, s_{2}\right) \mathrm{d} \sigma_{2}^{*}\left(s_{2}\right) \quad\left(\begin{array}{c}
(\text { by } 1): u_{1}\left(a^{\prime}+\varepsilon, s_{2}\right)=0 \\
\left.\forall s_{2}>1-a^{\prime}-\varepsilon\right)
\end{array}\right) \\
& =\int_{\left[0,1-s^{\prime \prime}\right]} v_{1}\left(a^{\prime}+\varepsilon, s_{2}\right) \mathrm{d} \sigma_{2}^{*}\left(s_{2}\right) \quad\left(\sigma_{2}^{*}\left(\left(1-a^{\prime}-\varepsilon, 1-s^{\prime \prime}\right]\right)=0\right) \\
> & \int_{\left[0,1-s^{\prime \prime}\right]} v_{1}\left(s^{\prime \prime}, s_{2}\right) \mathrm{d} \sigma_{2}^{*}\left(s_{2}\right) \quad\left(\begin{array}{c}
\left(\text { Assumption } 1(\text { iii }), a^{\prime}+\varepsilon>s^{\prime \prime}\right. \\
\text { and } \left.\sigma_{2}^{*}(0)<1\right)
\end{array}\right) \\
= & U_{1}\left(s^{\prime \prime}, \sigma_{2}^{*}\right) . \quad\left(\text { definition of } U_{1}\right)
\end{aligned}
$$

Thus, $U_{1}\left(\sigma_{1}^{*}, \sigma_{2}^{*}\right)>U_{1}\left(s^{\prime \prime}, \sigma_{2}^{*}\right)$ for all $s^{\prime \prime} \in\left(a^{\prime}-\varepsilon, a^{\prime}+\varepsilon\right)$. Therefore, an optimal strategy for player 1 cannot assign positive probability to any subset of $\left(a^{\prime}-\varepsilon, a^{\prime}+\varepsilon\right)$; that is, $\sigma_{1}^{*}\left(\left(a^{\prime}-\varepsilon, a^{\prime}+\varepsilon\right)\right)=0$; but this contradicts the assumption that $a^{\prime} \in A=$ $\operatorname{supp}\left(\sigma_{1}^{*}\right)$. Therefore, $a^{\prime} \in A \Longrightarrow 1-a^{\prime} \in B$.

Proof of Proposition 2. The assumptions that $A$ and $B$ are nonempty and balanced imply there is an integer $n \geq 1$ such that $A$ and $B$ both have $n$ distinct elements. The case of $n=1$ is obviously a PSE with balanced demands. For the remainder of the 
proof, suppose $n \geq 2$. The sets $A$ and $B$ may be written as $A \equiv\left\{a_{1}, a_{2}, \ldots, a_{n}\right\}$ and $B \equiv\left\{b_{1}, b_{2}, \ldots, b_{n}\right\}$, where $a_{1}<a_{2}<\cdots<a_{n}<1$ and $b_{1}<b_{2}<\cdots<b_{n}<1$. Balancedness implies $a_{1}=1-b_{n}$ and $b_{1}=1-a_{n}$; given $1 \notin A \cup B$, it follows that $a_{1}>0$ and $b_{1}>0$.

I now derive player 2's unique strategy that leaves player 1 willing to completely mix over $A$. Consider the following system of equations:

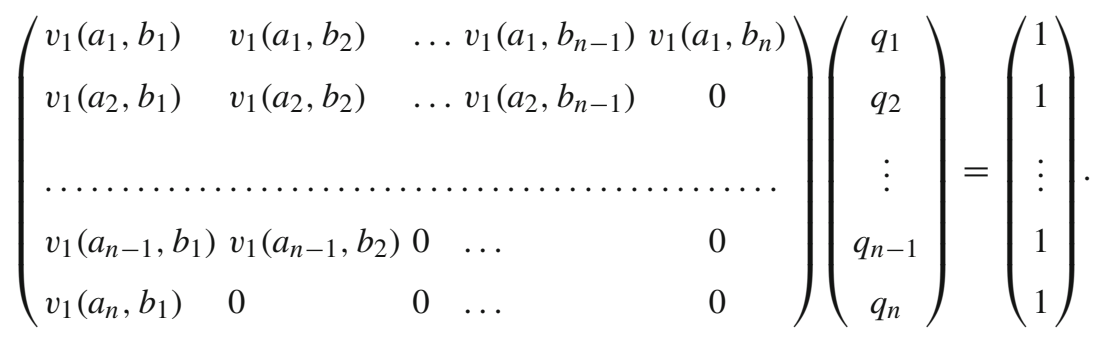

Because $a_{j}>0$ and $b_{j}>0 \forall j$, Assumption 1(iii) implies $v_{1}\left(a_{i}, b_{j}\right)>0 \forall i, j=$ $1, \ldots, n$. Denote by $V$ the $n \times n$ matrix in (12). The determinant of $V$ is nonzero: $|\operatorname{det}(V)|=\prod_{k=1}^{n} v_{1}\left(a_{n+1-k}, b_{k}\right)>0$. Thus, $V$ is invertible. Hence, there exists a unique vector $\mathbf{q}=\left(q_{1}, q_{2}, \ldots, q_{n}\right)$ that solves (12).

I claim q satisfies $q_{i}>0$ for $i=1, \ldots, n$. First, observe that (12) is equivalent to the following:

$$
1=\sum_{j=1}^{k} v_{1}\left(a_{n+1-k}, b_{j}\right) q_{j}, \quad k=1,2, \ldots, n
$$

I show by induction that $q_{j}>0$ for $j=1, \ldots, n$. Consider (13) for $k=1: 1=$ $v_{1}\left(a_{n}, b_{1}\right) q_{1}$, so $q_{1}>0$. Suppose it has been shown that $q_{j}>0$ for $j=1, \ldots, k$. Then, for $k+1$, Eq. (13) becomes

$$
\begin{aligned}
1 & =\sum_{j=1}^{k+1} v_{1}\left(a_{n+1-(k+1)}, b_{j}\right) q_{j} \\
& =\sum_{j=1}^{k} v_{1}\left(a_{n+1-(k+1)}, b_{j}\right) q_{j}+v_{1}\left(a_{n+1-(k+1)}, b_{k+1}\right) q_{k+1} \\
& <\sum_{j=1}^{k} v_{1}\left(a_{n+1-k}, b_{j}\right) q_{j}+v_{1}\left(a_{n+1-(k+1)}, b_{k+1}\right) q_{k+1} \\
& =1+v_{1}\left(a_{n+1-(k+1)}, b_{k+1}\right) q_{k+1},
\end{aligned}
$$

where (14) follows from Assumption 1(iii) and $a_{n+1-k}>a_{n+1-(k+1)}$; (15) follows from (13). Consequently, $v_{1}\left(a_{n+1-(k+1)}, b_{k+1}\right) q_{k+1}>0$, which in turn implies $q_{k+1}>0$. Therefore, $q_{j}>0$ for $j=1, \ldots, n$. Now for player 2 define the mixed 
strategy $\sigma_{2}^{*}$ as follows:

$$
\sigma_{2}^{*}\left(b_{j}\right)=q_{j} / \sum_{i=1}^{n} q_{i} \quad j=1,2, \ldots, n .
$$

Because $q_{j}>0$ for $j=1, \ldots, n$, it follows that $\sigma_{2}^{*}$ is completely mixed over $B$.

From (16) and (13) it follows that

$$
U_{1}\left(a, \sigma_{2}^{*}\right)=1 / \sum_{i=1}^{n} q_{i} \quad \forall a \in A .
$$

To see player 1 is willing to completely mix over $A$, it remains to show that player 1 finds demands outside $A$ are inferior to those in $A$. But this follows from exactly the same reasoning as in Example 1. For all $s>a_{n}$ disagreement is certain, so $U_{1}\left(s, \sigma_{2}^{*}\right)=$ $0 \forall s>a_{n}$. Because $A$ and $B$ are balanced, the payoff $U_{1}\left(s, \sigma_{2}^{*}\right)$ is strictly increasing in $s$ over the interval $\left[0, a_{1}\right]$ and over each of the intervals $\left(a_{j-1}, a_{j}\right], j=2, \ldots, n$; over each of these intervals $U_{1}\left(s, \sigma_{2}^{*}\right)$ is maximized at the right endpoint, which is itself an element of $A$. Thus, $U_{1}\left(s, \sigma_{2}^{*}\right)<1 / \sum_{i=1}^{n} q_{i} \forall s \notin A$.

The foregoing analysis shows that under the assumptions of the proposition there exists a unique strategy $\sigma_{2}^{*} \in \Sigma$ that is completely mixed over $B$ and leaves player 1 willing to play any strategy that is mixed over $A$. A similar argument shows there exists a unique strategy $\sigma_{1}^{*} \in \Sigma$ that is completely mixed over $A$ and leaves player 2 willing to play any strategy that is mixed over $B$. Then $\left(\sigma_{1}^{*}, \sigma_{2}^{*}\right)$ is the unique equilibrium in which player 1 completely mixes over $A$ and player 2 completely mixes over $B$.

Proof of Proposition 3. For convenience, I gather three facts from probability theory. Let $X$ be a nonempty compact subset of a Euclidean space and let $\mathcal{A}_{X}$ be the Borel sigma-algebra on $X$. If a sequence of probability measures $\left(\mu^{n}\right)_{n}$ on $\left(X, \mathcal{A}_{X}\right)$ converges to a limit $\mu^{0}$ in the topology of weak convergence, I denote this by $\mu^{n} \stackrel{w}{\longrightarrow} \mu^{0}$. In the statement of these facts, $\left(\mu^{n}\right)_{n}$ and $\mu^{0}$ are probability measures on $\left(X, \mathcal{A}_{X}\right)$. [References are as follows: Fact 1, Billingsley (1999, Ch. 1.5), Fact 2, Billingsley (1999, p. 24), and Fact 3, Loève (1977, Ch. 12.1.)]

Fact 1 For any sequence $\left(\mu^{n}\right)_{n}$, there exists a subsequence $\left(\mu^{n_{k}}\right)_{k}$ and a probability measure $\mu^{0}$ such that $\mu^{n_{k}} \stackrel{w}{\longrightarrow} \mu^{0}$.

Fact 2 If $h: X \rightarrow \mathbb{R}$ is upper-semicontinuous and if $\mu^{n} \stackrel{w}{\longrightarrow} \mu^{0}$, then $\int h d \mu^{0} \geq \lim \sup \int h d \mu^{n}$.

Fact 3 Suppose $h: X \rightarrow \mathbb{R}$ and let $D$ denote the set of points of discontinuity of $h$. If $\mu^{0}(D)=0$ and $\mu^{n} \stackrel{w}{\longrightarrow} \mu^{0}$, then $\lim \int h d \mu^{n}$ exists and $\lim \int h d \mu^{n}=$ $\int h d \mu^{0}$.

Define $\underline{a} \equiv \min \{a \mid a \in A\}, \bar{a} \equiv \max \{a \mid a \in A\}, \underline{b} \equiv \min \{b \mid b \in B\}$, and $\bar{b} \equiv \max \{\bar{b} \mid b \in B\}$. Next define $I_{A} \equiv[\underline{a}, \bar{a}]$ and $I_{B} \equiv[\underline{b}, \bar{b}]$. Because $1 \notin A \cup B$, balancedness implies $\underline{a}>0$ and $\underline{b}>0$. Assumption 2 ensures $v_{1}$ and $v_{2}$ are continuous on $I_{A} \times I_{B}$. Let $\left(a_{k}\right)_{k=1}^{\infty}$ be a countable dense subset of $A$ with $a_{1}=\underline{a}$ and $a_{2}=\bar{a}$. Next define $\left(b_{k}\right)_{k=1}^{\infty}$ by $b_{k}=1-a_{k}$ for all $k$. Because $A$ and $B$ are balanced, $\left(b_{k}\right)_{k}$ 
is a dense subset of $B$. Next for every integer $n \geq 1$, define $A^{n} \equiv\left\{a_{1}, \ldots, a_{n}\right\}$ and $B^{n} \equiv\left\{b_{1}, \ldots, b_{n}\right\}$.

By Proposition 2, for each $n \geq 1$ there exists a unique equilibrium $\left(\sigma_{1}^{n}, \sigma_{2}^{n}\right)$ such that $\sigma_{1}^{n}$ is completely mixed over $A^{n}$ and $\sigma_{2}^{n}$ is completely mixed over $B^{n}$. Because $S$ is compact, Fact 1 implies there exists a subsequence of $\left(\sigma_{1}^{n}, \sigma_{2}^{n}\right)_{n}$ that converges weakly to a limit $\left(\sigma_{1}^{0}, \sigma_{2}^{0}\right)$, where $\sigma_{1}^{0}$ and $\sigma_{2}^{0}$ are probability measures on $S$. Without loss of generality and to simplify notation, I take the original sequence $\left(\sigma_{1}^{n}, \sigma_{2}^{n}\right)_{n}$ to converge to $\left(\sigma_{1}^{0}, \sigma_{2}^{0}\right)$. Note that the supports of $\left(\sigma_{1}^{n}\right)_{n}$ and $\sigma_{1}^{0}$ are contained in $I_{A}$ and the supports of $\left(\sigma_{2}^{n}\right)_{n}$ and $\sigma_{2}^{0}$ are contained in $I_{B}$.

To prove the proposition, it must be shown that

I. $\left(\sigma_{1}^{0}, \sigma_{2}^{0}\right)$ is an equilibrium and

II. $\operatorname{supp}\left(\sigma_{1}^{0}\right)=A$ and $\operatorname{supp}\left(\sigma_{2}^{0}\right)=B$.

Subproof of I. Consider player 1. I will show

$$
U_{1}\left(\sigma_{1}^{0}, \sigma_{2}^{0}\right) \geq U_{1}\left(s_{1}, \sigma_{2}^{0}\right) \quad \forall s_{1} \in S
$$

The corresponding condition for player 2 is proven analogously. There are two cases: (i) $1-s_{1}$ is not an atom of $\sigma_{2}^{0}$ (i.e., $\sigma_{2}^{0}\left(1-s_{1}\right)=0$ ) and (ii) $1-s_{1}$ is an atom of $\sigma_{2}^{0}$ (i.e., $\left.\sigma_{2}^{0}\left(1-s_{1}\right)>0\right)$.

Consider case (i): $1-s_{1}$ is not an atom of $\sigma_{2}^{0}$. Then, for every $n$,

$$
U_{1}\left(\sigma_{1}^{n}, \sigma_{2}^{n}\right) \geq U_{1}\left(s_{1}, \sigma_{2}^{n}\right)
$$

because $\left(\sigma_{1}^{n}, \sigma_{2}^{n}\right)$ is an equilibrium. Furthermore, $u_{1}$ is upper-semicontinuous on $I_{A} \times$ $I_{B},{ }^{11}$ and on $I_{B}$ the only discontinuity of $u_{1}\left(s_{1}, \cdot\right)$ occurs at $s_{2}=1-s_{1}$, which is not an atom of $\sigma_{2}^{0}$. Therefore,

$$
\begin{aligned}
U_{1}\left(\sigma_{1}^{0}, \sigma_{2}^{0}\right) & \geq \lim \sup U_{1}\left(\sigma_{1}^{n}, \sigma_{2}^{n}\right) \quad(\text { Fact } 2) \\
& \geq \lim \sup U_{1}\left(s_{1}, \sigma_{2}^{n}\right) \quad(\text { by }(18)) \\
& =\lim U_{1}\left(s_{1}, \sigma_{2}^{n}\right) \quad(\text { Fact } 3) \\
& =U_{1}\left(s_{1}, \sigma_{2}^{0}\right), \quad(\text { Fact } 3)
\end{aligned}
$$

thus proving (17) holds in case (i).

Now consider case (ii): $1-s_{1}$ is an atom of $\sigma_{2}^{0}$. I prove by contradiction that (17) must hold in this case too. Suppose to the contrary that (17) is not satisfied; that is,

11 A function $h: X \rightarrow \mathbb{R}$ is upper-semicontinuous if for any $\alpha \in \mathbb{R}$ the set $\{x \in X \mid h(x)<\alpha\}$ is open in $X$. Now, for any $\alpha>0$,

$$
\begin{aligned}
\left\{\left(s_{1}, s_{2}\right) \in I_{A} \times I_{B} \mid u_{1}\left(s_{1}, s_{2}\right)<\alpha\right\}= & \left\{\left(s_{1}, s_{2}\right) \in I_{A} \times I_{B} \mid v_{1}\left(s_{1}, s_{2}\right)<\alpha\right\} \\
& \cup\left\{\left(s_{1}, s_{2}\right) \in I_{A} \times I_{B} \mid s_{1}+s_{2}>1\right\} .
\end{aligned}
$$

Because $(0,0) \notin I_{A} \times I_{B}, v_{1}$ is continuous on $I_{A} \times I_{B}$; therefore, both sets on the right-hand side of (*) are open in $I_{A} \times I_{B}$, and so too is their union. And for $\alpha \leq 0$, the left-hand side of (*) is just $I_{A} \times I_{B}$. Thus, $u_{1}$ is upper-semicontinuous on $I_{A} \times I_{B}$. 
$U_{1}\left(s_{1}, \sigma_{2}^{0}\right)>U_{1}\left(\sigma_{1}^{0}, \sigma_{2}^{0}\right)$. Let $\left(s_{1}^{k}\right)_{k}$ be a sequence such that $s_{1}^{k} \rightarrow s_{1}$ and for any $k$, $s_{1}^{k}<s_{1}$ and $1-s_{1}^{k}$ is not an atom of $\sigma_{2}^{0}$. Then for all $s_{2} \in S, u_{1}\left(s_{1}^{k}, s_{2}\right) \rightarrow u_{1}\left(s_{1}, s_{2}\right)$ as $k \rightarrow \infty$. Therefore, by the Bounded Convergence Theorem [Chung (1974, p. 42)], $U_{1}\left(s_{1}^{k}, \sigma_{2}^{0}\right) \rightarrow U_{1}\left(s_{1}, \sigma_{2}^{0}\right)$. Consequently, if $U_{1}\left(s_{1}, \sigma_{2}^{0}\right)>U_{1}\left(\sigma_{1}^{0}, \sigma_{2}^{0}\right)$, then there exists $k^{\prime}$ such that $s_{1}^{k^{\prime}}<s_{1}, 1-s_{1}^{k^{\prime}}$ is not an atom of $\sigma_{2}^{0}$, and

$$
U_{1}\left(s_{1}^{k^{\prime}}, \sigma_{2}^{0}\right)>U_{1}\left(\sigma_{1}^{0}, \sigma_{2}^{0}\right)
$$

but (19) contradicts case (i). Hence, it must be that (17) holds also in case (ii).

Analogous reasoning shows $U_{2}\left(\sigma_{1}^{0}, \sigma_{2}^{0}\right) \geq U_{1}\left(\sigma_{1}^{0}, s_{2}\right)$ for all $s_{2} \in S$. Thus, part I is proven. A.

Subproof of II. This is shown with two steps: (i) $\operatorname{supp}\left(\sigma_{1}^{0}\right) \subset A$ and (ii) $\operatorname{supp}\left(\sigma_{1}^{0}\right) \supset$

Consider step (i). Let $s_{1} \in S$ such that $s_{1} \notin A$. Because $A$ is closed, there exists $\varepsilon>0$ such that $\left(s_{1}-\varepsilon, s_{1}+\varepsilon\right) \cap A=\varnothing$ and such that $s_{1}-\varepsilon$ and $s_{1}+\varepsilon$ are not atoms of $\sigma_{1}^{0}$. Therefore,

$$
\sigma_{1}^{0}\left(\left(s_{1}-\varepsilon, s_{1}+\varepsilon\right)\right)=\lim \sigma_{1}^{n}\left(\left(s_{1}-\varepsilon, s_{1}+\varepsilon\right)\right)=0,
$$

where the first equality follows by weak convergence and the second because $\sigma_{1}^{n}\left(\left(s_{1}-\right.\right.$ $\left.\left.\varepsilon, s_{1}+\varepsilon\right)\right)=0$ for every $n$. Thus, $s_{1} \notin \operatorname{supp}\left(\sigma_{1}^{0}\right)$, so $\operatorname{supp}\left(\sigma_{1}^{0}\right) \subset A$.

To establish step (ii), suppose to the contrary that $\exists a^{0} \in A$ such that $a^{0} \notin \operatorname{supp}\left(\sigma_{1}^{0}\right)$. Because $1 \notin A \cup B$ and $A$ and $B$ are balanced, it follows that $0<a^{0}<1$; therefore, $\exists \varepsilon^{0}>0$ such that $0<a^{0}-\varepsilon^{0}<a^{0}+\varepsilon<1$ and $\sigma_{1}^{0}\left(\left(a^{0}-\varepsilon^{0}, a^{0}+\varepsilon^{0}\right)\right)=0$. Because $\bigcup A_{k}$ is dense in $A, \exists a^{\prime} \in \bigcup A_{k}$ and $\varepsilon>0$ such that $\left(a^{\prime}-\varepsilon, a^{\prime}+\varepsilon\right) \subset\left(a^{0}-\varepsilon^{0}, a^{0}+\varepsilon^{0}\right)$ and neither $a^{\prime}-\varepsilon$ nor $a^{\prime}+\varepsilon$ is an atom of $\sigma_{1}^{0}$; therefore, because $\sigma_{1}^{n} \stackrel{w}{\longrightarrow} \sigma_{1}^{0}$, it follows that

$$
\lim \sigma_{1}^{n}\left(\left(a^{\prime}-\varepsilon, a^{\prime}+\varepsilon\right)\right)=\sigma_{1}^{0}\left(\left(a^{\prime}-\varepsilon, a^{\prime}+\varepsilon\right)\right) \leq \sigma_{1}^{0}\left(\left(a^{0}-\varepsilon^{0}, a^{0}+\varepsilon^{0}\right)\right)=0 .
$$

Because the $A_{n}$ sets are nested, there exists some $n^{\prime} \geq 2$ such that $a^{\prime} \in A_{n}$ for all $n \geq n^{\prime}$. Observe that for all $n \geq n^{\prime}$,

$$
\begin{aligned}
0<v_{2}(\bar{a}, 1-\bar{a}) \leq & U_{2}\left(\sigma_{1}^{n}, 1-\bar{a}\right) \quad(\text { Assumption 1(iii)) } \\
= & U_{2}\left(\sigma_{1}^{n}, \sigma_{2}^{n}\right) \quad\left(1-\bar{a} \in B^{n} \forall n \geq 2\right) \\
= & \int_{\left[\underline{a}, a^{\prime}\right]} v_{2}\left(s_{1}, 1-a^{\prime}\right) \mathrm{d} \sigma_{1}^{n}\left(s_{1}\right) \\
& \left(1-a^{\prime} \in B^{n} \forall n \geq 2, \text { and def. of } U_{2}\right) \\
\leq & \sigma_{1}^{n}\left(\left[\underline{a}, a^{\prime}\right]\right) v_{2}\left(\underline{a}, 1-a^{\prime}\right), \quad \text { (Assumption 1(iii)) }
\end{aligned}
$$


from which it follows that

$$
\sigma_{1}^{n}\left(\left[\underline{a}, a^{\prime}\right]\right) \geq \frac{v_{2}(\bar{a}, 1-\bar{a})}{v_{2}\left(\underline{a}, 1-a^{\prime}\right)}>0 \quad \forall n \geq n^{\prime} .
$$

Now define

$$
\Delta \equiv \min _{\underline{a} \leq s_{1} \leq a^{\prime}-\varepsilon}\left\{v_{2}\left(s_{1}, 1-a^{\prime}+\varepsilon\right)-v_{2}\left(s_{1}, 1-a^{\prime}\right)\right\}
$$

Assumptions 1(iii) and 2 imply $\Delta>0$. Next I show player 2 does better to demand $1-a^{\prime}+\varepsilon$ than to play $\sigma_{2}^{n}$ when player 1 plays $\sigma_{1}^{n}$. Suppose $n \geq n^{\prime}$; then

$$
\begin{aligned}
U_{2}\left(\sigma_{1}^{n}, 1-a^{\prime}+\right. & \varepsilon)-U_{2}\left(\sigma_{1}^{n}, \sigma_{2}^{n}\right)=U_{2}\left(\sigma_{1}^{n}, 1-a^{\prime}+\varepsilon\right)-U_{2}\left(\sigma_{1}^{n}, 1-a^{\prime}\right) \\
= & \int_{\left[\underline{a}, a^{\prime}-\varepsilon\right]}\left[v_{2}\left(s_{1}, 1-a^{\prime}+\varepsilon\right)-v_{2}\left(s_{1}, 1-a^{\prime}\right)\right] \mathrm{d} \sigma_{1}^{n}\left(s_{1}\right) \\
& -\int_{\left(a^{\prime}-\varepsilon, a^{\prime}\right]} v_{2}\left(s_{1}, 1-a^{\prime}\right) \mathrm{d} \sigma_{1}^{n}\left(s_{1}\right) \\
\geq & \Delta \times \sigma_{1}^{n}\left(\left[\underline{a}, a^{\prime}-\varepsilon\right]\right)-v_{2}\left(a^{\prime}-\varepsilon, 1-a^{\prime}\right) \sigma_{1}^{n}\left(\left(a^{\prime}-\varepsilon, a^{\prime}\right]\right) \\
= & \Delta \times \sigma_{1}^{n}\left(\left[\underline{a}, a^{\prime}\right]\right)-(\Delta 4) \\
\geq & \Delta \times \frac{v_{2}(\bar{a}, 1-\bar{a})}{v_{2}\left(\underline{a}, 1-a^{\prime}\right)}-\left(\Delta+v_{2}\left(a^{\prime}-\varepsilon, 1-a^{\prime}\right)\right) \sigma_{1}^{n}\left(\left(a^{\prime}-\varepsilon, a^{\prime}\right]\right) \\
& \\
\longrightarrow & \Delta \times \frac{v_{2}(\bar{a}, 1-\bar{a})}{v_{2}\left(\underline{a}, 1-a^{\prime}\right)} \text { as } n \rightarrow \sigma_{1}^{\prime}\left(\left(a^{\prime}-\varepsilon, a^{\prime}\right]\right) \\
> & 0 .
\end{aligned}
$$

Here (22) follows because $\left(\sigma_{1}^{n}, \sigma_{2}^{n}\right)$ is an equilibrium with $\sigma_{2}^{n}\left(1-a^{\prime}\right)>0$; (23) follows from the definitions of $U_{2}$ and $u_{2}$; (24) follows from the definitions of $\Delta$ and Assumption 1(iii); (25) follows from (21); (26) follows from (20); and (27) follows from (21) and $\Delta>0$. Thus, for all $n$ sufficiently large, $U_{2}\left(\sigma_{1}^{n}, 1-a^{\prime}+\varepsilon\right)>U_{2}\left(\sigma_{1}^{n}, \sigma_{2}^{n}\right)$, which contradicts $\left(\sigma_{1}^{n}, \sigma_{2}^{n}\right)$ being an equilibrium. Hence, it must be the case that $\operatorname{supp}\left(\sigma_{1}^{0}\right) \supset A$. Steps (i) and (ii) now establish that $\operatorname{supp}\left(\sigma_{1}^{0}\right)=A$; that is, $\sigma_{1}^{0}$ is completely mixed over $A$.

Analogous reasoning shows $\sigma_{2}^{0}$ is completely mixed over $B$. Thus, part II is proven. 
Proof of Proposition 4. Let $\sigma_{i}^{*}$ denote the mixed strategy associated with the $\operatorname{cdf} F_{i}$ given in the proposition, $i=1,2$. Note that a point $s \in S$ is an element of the support of $\sigma_{i}^{*}$, and so too $F_{i}$, if and only if $\forall \varepsilon>0, F_{i}(s+\varepsilon)-F_{i}(s-\varepsilon)>0$.

Step 1: $\operatorname{supp}\left(\sigma_{1}^{*}\right)=A$ and $\operatorname{supp}\left(\sigma_{2}^{*}\right)=B$.

I show $A=\operatorname{supp}\left(F_{1}\right)$; the proof that $B=\operatorname{supp}\left(F_{2}\right)$ is analogous. First suppose $s \notin A$. Because $A$ is closed, $\exists \varepsilon^{\prime}>0$ such that $\left(s-2 \varepsilon^{\prime}, s+2 \varepsilon^{\prime}\right) \cap A=\varnothing$, implying $F_{1}\left(s+\varepsilon^{\prime}\right)=F_{1}\left(s-\varepsilon^{\prime}\right)$, in turn implying $F_{1}\left(s+\varepsilon^{\prime}\right)-F_{1}\left(s-\varepsilon^{\prime}\right)=0$. Thus, $s \notin \operatorname{supp}\left(F_{1}\right)$, so $\operatorname{supp}\left(\sigma_{1}^{0}\right) \subset A$.

Now suppose $a \in A$. Either $a$ is an isolated point of $A$ or it is not. If $a$ is an isolated point of $A$, then it is a jump point of $F_{1}$ [Chung $\left(1974\right.$, p. 10)] so that $\lim _{s \uparrow a} F_{1}(s)<$ $F_{1}(a)$. Therefore, $\forall \varepsilon>0, F_{1}(a+\varepsilon)-F_{1}(a-\varepsilon) \geq F_{1}(a)-\lim _{s \uparrow a} F_{1}(s)>0$, implying $a \in \operatorname{supp}\left(F_{1}\right)$. Now suppose $a \in A$ is not an isolated point of $A$. Then $\forall \varepsilon>0, \exists a(\varepsilon) \in A$ such that $a(\varepsilon) \neq a$ and $|a-a(\varepsilon)|<\varepsilon$. Consequently,

$$
F_{1}(a+\varepsilon)-F_{1}(a-\varepsilon) \geq\left|F_{1}(a)-F_{1}(a(\varepsilon))\right|=\left|\frac{w_{2}(\underline{b})}{w_{2}(1-a)}-\frac{w_{2}(\underline{b})}{w_{2}(1-a(\varepsilon))}\right|>0,
$$

where the inequality follows because $w_{2}$ is strictly increasing and $a \neq a(\varepsilon)$. Therefore, $a \in \operatorname{supp}\left(F_{1}\right), \operatorname{so} \operatorname{supp}\left(F_{1}\right) \supset A$. The foregoing cases $\operatorname{show} \operatorname{supp}\left(F_{1}\right)=A$.

Step 2: if $A$ and $B$ are balanced, then $\left(\sigma_{1}^{*}, \sigma_{2}^{*}\right)$ is an equilibrium.

Suppose $A$ and $B$ are balanced. Because $A$ and $B$ are nonempty closed sets such that $1 \notin A \cup B$, balancedness implies $\underline{a}=1-\bar{b}>0$ and $\underline{b}=1-\bar{a}>0$. Consider player 1's situation. For player 1,

$$
U_{1}\left(a, \sigma_{2}^{*}\right)=w_{1}(a) F_{2}(1-a)=w_{1}(a)\left(\frac{w_{1}(\underline{a})}{w_{1}(1-(1-a))}\right)=w_{1}(\underline{a}) \quad \forall a \in A .
$$

To show $\sigma_{1}^{*}$ is a best response for player 1 to $\sigma_{2}^{*}$ being played by player 2 , I next show

$$
U_{1}\left(s_{1}, \sigma_{2}^{*}\right)<w_{1}(\underline{a}) \quad \forall s_{1} \notin A .
$$

There are two cases to consider. First, suppose $s_{1}=s^{\prime}>\bar{a}$. Then $\forall s_{2} \in B, s^{\prime}+s_{2} \geq$ $s^{\prime}+\underline{b}>\bar{a}+\underline{b}=1$, so $U_{1}\left(s^{\prime}, \sigma_{2}^{*}\right)=0<w_{1}(\underline{a})$. Second, suppose $s_{1}=s^{\prime}$ such that $s^{\prime} \notin A$ and $s^{\prime}<\bar{a}$. Define $a^{\prime} \equiv \min \left\{a \in A \mid a>s^{\prime}\right\}$. Then $a^{\prime} \in A, a^{\prime}>s^{\prime}$, and $\left[s^{\prime}, a^{\prime}\right) \cap A=\varnothing$; so balancedness of $A$ and $B$ implies $\left(1-a^{\prime}, 1-s^{\prime}\right] \cap B=\varnothing$. Therefore, $F_{2}\left(1-s^{\prime}\right)=F_{2}\left(1-a^{\prime}\right)>0$, implying

$$
\begin{aligned}
w_{1}(\underline{a}) & =U_{1}\left(a^{\prime}, \sigma_{2}^{*}\right)=w_{1}\left(a^{\prime}\right) F_{2}\left(1-a^{\prime}\right) \\
& >w_{1}\left(s^{\prime}\right) F_{2}\left(1-a^{\prime}\right)=w_{1}\left(s^{\prime}\right) F_{2}\left(1-s^{\prime}\right) \\
& =U_{1}\left(s^{\prime}, \sigma_{2}^{*}\right) .
\end{aligned}
$$

The two cases just discussed show (28) is satisfied. Thus, $\sigma_{1}^{*}$ is a best response for player 1 to $\sigma_{2}^{*}$ being played by player 2 ; similarly, $\sigma_{2}^{*}$ can be shown to be a best response for player 2 to $\sigma_{1}^{*}$ being played by player 1 . Thus, $\left(\sigma_{1}^{*}, \sigma_{2}^{*}\right)$ is an equilibrium in which $\sigma_{1}^{*}$ completely mixes over $A$ and $\sigma_{2}^{*}$ completely mixes over $B$. 
Step 3: $\left(\sigma_{1}^{*}, \sigma_{2}^{*}\right)$ is the unique equilibrium in which player 1 completely mixes over $A$ and player 2 completely mixes over $B$.

Suppose $\left(\mu_{1}^{*}, \mu_{2}^{*}\right)$ is any equilibrium in which $\mu_{1}^{*}$ completely mixes over $A$ and $\mu_{2}^{*}$ completely mixes over $B$. Let $G$ be the cdf associated with $\mu_{2}^{*}$. Recall that a probability measure uniquely determines an associated cdf, and vice versa. First, I show $G(b)=$ $F_{2}(b) \forall b \in B$. Observe that $w_{1}(\underline{a})=U_{1}\left(\underline{a}, \mu_{2}^{*}\right)=U_{1}\left(a, \mu_{2}^{*}\right)=w_{1}(a) G(1-a)$ $\forall a \in A$. Because $A$ and $B$ are balanced, with the change of variables $b=1-a$, I rewrite this condition as

$$
G(b)=\frac{w_{1}(\underline{a})}{w_{1}(1-b)} \quad \forall b \in B ;
$$

that is, $G(b)=F_{2}(b) \forall b \in B$. Because $F_{2}$ and $G$ agree on $B, \sigma_{2}^{*}$ and $\mu_{2}^{*}$ agree on all measurable subsets of $B$; in particular, $\mu_{2}^{*}(B)=\sigma_{2}^{*}(B)=1$. Because $\mu_{2}^{*}$ is a probability measure, it therefore assigns no mass outside of $B$, agreeing with $\sigma_{2}^{*}$. It follows that the probability measures $\sigma_{2}^{*}$ and $\mu_{2}^{*}$ are identical. A symmetric argument shows that $\mu_{1}^{*}$ is identical to $\sigma_{1}^{*}$.

Example 8 [A continuous version of Roth's example (Roth 1985)] Suppose $v_{1}$ and $v_{2}$ are given by (3). Let $a \in(0,1 / 2)$ be given and define $A \equiv[a, 1 / 2]$ and $B \equiv$ $[1 / 2,1-a]$. The sets $A$ and $B$ are balanced. Consider strategies for players 1 and 2 given by the cdfs $F_{1}$ and $F_{2}$, respectively. Note that for $s_{1}, s_{2}$ with $s_{1}+s_{2} \leq 1$ we have $v_{i}\left(s_{1}, s_{2}\right)=\frac{1}{2}\left(1+s_{i}-s_{j}\right), j \neq i, i=1,2$. Now the expected payoff for player 1 using $s_{1} \in A$ is given by

$$
\begin{aligned}
U_{1}\left(s_{1}, F_{2}\right) & =\frac{1}{2} F_{2}(1 / 2)\left(1+s_{1}-\frac{1}{2}\right)+\frac{1}{2} \int_{1 / 2}^{1-s_{1}}\left(1+s_{1}-s_{2}\right) f_{2}\left(s_{2}\right) \mathrm{d} s_{2} \\
& =s_{1} F_{2}\left(1-s_{1}\right)+\frac{1}{2} \int_{1 / 2}^{1-s_{1}} F_{2}\left(s_{2}\right) \mathrm{d} s_{2} . \quad \text { (integration by parts) }
\end{aligned}
$$

If player 1 is to be willing to randomize over [ $a, 1 / 2]$, it must be that over this interval $U_{1}\left(s_{1}, F_{2}\right)$ is constant with respect to $s_{1}$ Therefore, we require that for $s_{1} \in(a, 1 / 2)$,

$$
0=\frac{\partial U_{1}}{\partial s_{1}}=\frac{1}{2} F_{2}\left(1-s_{1}\right)-s_{1} f_{2}\left(1-s_{1}\right),
$$

a differential equation that may be rewritten, with the change of variables $s_{2}=1-s_{1}$, as

$$
\frac{f_{2}\left(s_{2}\right)}{F_{2}\left(s_{2}\right)}=\frac{1}{2\left(1-s_{2}\right)} .
$$


Accounting for the boundary condition $F_{2}(1-a)=1$, the solution to this differential equation is found to be

$$
F_{2}\left(s_{2}\right)=\sqrt{\frac{a}{1-s_{2}}}, \quad \forall s_{2} \in[1 / 2,1-a] .
$$

Similarly, player 1's equilibrium strategy is found to be described by the cdf

$$
F_{1}\left(s_{1}\right)=\frac{1}{\sqrt{2\left(1-s_{1}\right)}}, \quad \forall s_{1} \in[a, 1 / 2] .
$$

Thus, $\left(F_{1}, F_{2}\right)$ constitutes an equilibrium in which player 1 completely mixes over $[a, 1 / 2]$ and player 2 completely mixes over $[1 / 2,1-a]$. The probability of disagreement is calculated as

$$
\begin{aligned}
\operatorname{Pr}(\text { disagreement }) & =\int_{a}^{1 / 2}\left[1-F_{2}\left(1-s_{1}\right)\right] f_{1}\left(s_{1}\right) \mathrm{d} s_{1} \\
& =\int_{a}^{1 / 2} \frac{\mathrm{d} s_{1}}{2 \sqrt{2}\left(1-s_{1}\right)^{3 / 2}}-\frac{\sqrt{a}}{2 \sqrt{2}} \int_{a}^{1 / 2} \frac{\mathrm{d} s_{1}}{\sqrt{s_{1}}\left(1-s_{1}\right)^{3 / 2}} \\
& =1-\frac{\sqrt{a}+\sqrt{1-a}}{\sqrt{2}},
\end{aligned}
$$

which decreases from 0.2929 to 0 as $a$ increases from 0 to $1 / 2$. For all values of $a \in[0,1 / 2)$ this probability of disagreement is less than in Roth's discrete example, Example 2 above.

Proof of Proposition 5. First suppose $1 \in A$ and $1 \notin B$. Because $1 \notin B, \exists \varepsilon>0$ such that $(1-2 \varepsilon, 1] \cap B=\varnothing$. Therefore, if player 1 announces demand $\varepsilon$, then players' demands are sure to be compatible, so $U_{1}\left(\varepsilon, \sigma_{2}^{*}\right)=\varepsilon$. Because $\left(\sigma_{1}^{*}, \sigma_{2}^{*}\right)$ is an equilibrium, it follows that $U_{1}\left(\sigma_{1}^{*}, \sigma_{2}^{*}\right) \geq U_{1}\left(\varepsilon, \sigma_{2}^{*}\right)=\varepsilon>0$. Because $1 \in A, \exists\left(s_{1}^{k}\right)_{k} \subset S$ such that $s_{1}^{k} \rightarrow 1, s_{1}^{k}<1$, and $U_{1}\left(s_{1}^{k}, \sigma_{2}^{*}\right)=U_{1}\left(\sigma_{1}^{*}, \sigma_{2}^{*}\right)$ for each $k$. Therefore,

$$
0<U_{1}\left(\varepsilon, \sigma_{2}^{*}\right) \leq \lim U_{1}\left(s_{1}^{k}, \sigma_{2}^{*}\right)=\lim s_{1}^{k} F_{2}\left(1-s_{1}^{k}\right)=1 \times F_{2}(0),
$$

where the last equality follows from right-continuity of $F_{2}$. Thus, $F_{2}(0)>0$; that is, 0 is an atom of $F_{2}$. Because 0 is an atom of $F_{2}$, it follows that $U_{2}\left(\sigma_{1}^{*}, \sigma_{2}^{*}\right)=U_{2}\left(\sigma_{1}^{*}, 0\right)=0$, which in turn implies that player 1 demands 1 with probability 1 . To see this, observe that

$$
0=U_{2}\left(\sigma_{1}^{*}, \sigma_{2}^{*}\right) \geq U_{2}\left(\sigma_{1}^{*}, s\right)=s F_{1}(1-s) \geq 0 \quad \forall s \in(0,1]
$$

implying $F_{1}(s)=0$ for all $s<1$; hence, $\sigma_{1}^{*}(1)=1$. An analogous argument proves part 2. 
Finally suppose $1 \in A \cap B$. If $\sigma_{2}^{*}(1)=1$ the proof is done; so suppose $\sigma_{2}^{*}(1)<1$. Then $\exists \varepsilon>0$ such that $\sigma_{2}^{*}((1-2 \varepsilon, 1])<1$. If player 1 demands $\varepsilon$, then

$$
\left.U_{1}\left(\varepsilon, \sigma_{2}^{*}\right)=\varepsilon F_{2}(1-\varepsilon) \geq \varepsilon F_{2}(1-2 \varepsilon)=\varepsilon(1-\sigma(1-2 \varepsilon, 1])\right)>0
$$

Consequently, it must be that player 1's equilibrium payoff is positive, $U_{1}\left(\sigma_{1}^{*}, \sigma_{2}^{*}\right)>0$. The proof is completed as for part 1 , showing that 0 must be an atom of $\sigma_{2}^{*}$, which implies that player 2's equilibrium payoff is 0 , and thus player 1 demands 1 for sure. The details are omitted.

\section{References}

Anbarci, N.: Divide-the-dollar game revisited. Theory Decis 50, 295-304 (2001)

Baye, M.R., Kovenock, D., de Vries, C.: The solution to the Tullock rent-seeking game when $R>2$ : mixed-strategy equilibria and mean dissipation rates. Public Choice 81, 363-380 (1996)

Baye, M.R., Kovenock, D., de Vries, C.: The all-pay auction with complete information. Econ Theory 8 , 291-305 (1996)

Billingsley, P.: Convergence of Probability Measures, 2nd edn. New York: Wiley (1999)

Binmore, K.: Nash bargaining theory II. In: Binmore, K., Dasgupta, P. (eds.) The Economics of Bargaining, chap. 4,Oxford: Blackwell (1987)

Binmore, K., Rubinstein, A., Wolinsky, A.: The Nash bargaining solution in economic modelling. Rand J Econ 17, 176-188 (1986)

Camerer, C.: Behavioral Game Theory: Experiments in Strategic Interaction. Princeton: Princeton University Press (2003)

Carlsson, H.: A bargaining model where parties make errors. Econometrica 59, 1487-1496 (1991)

Chung, K.L.: A Course in Probability Theory, 2nd edn. New York: Academic Press (1974)

Crawford, V.P.: A theory of disagreement in bargaining. Econometrica 50, 607-638 (1982)

Dasgupta, P., Maskin, E.: The existence of equilibrium in discontinuous economic games, I: theory. Rev Econ Stud 53, 1-26 (1986)

Ellingsen, T., Miettinen, T.: Commitment and conflict in bilateral bargaining. Am Econ Rev 98, 1629-1635 (2008)

Fu, H.: Mixed-strategy equilibria and strong purification for games with private and public information. Econ Theory 37, 521-532 (2008)

Fudenberg, D., Tirole, J.: Game Theory. Cambridge: MIT Press (1991)

Harsanyi, J.: Games with randomly disturbed payoffs: a new rationale for mixed-strategy equilibrium points. Int J Game Theory 2, 1-23 (1973)

Kahn, M.A., Sun, Y.: Pure strategies in games with private information. J Math Econ 24, 633-653 (1995)

Loève, M. : Probability Theory I. New York: Springer (1977)

Milgrom, P.R., Weber, R.J.: Distribtuional strategies for games with incomplete information. Math Oper Res 10, 619-632 (1985)

Nash, J.F.: The bargaining problem. Econometrica 18, 155-162 (1950)

Nash, J.F.: Two-person cooperative games. Econometrica 21, 128-140 (1953)

Palfrey, T.R., Rosenthal, H.: Participation and the provision of discrete public goods: a strategic analysis. J Public Econ 24, 171-193 (1984)

Radner, R., Rosenthal, R.: Private information and pure-strategey equilibria. Math Oper Res 7, 401-409 (1982)

Robles, J.: Evolution, bargaining, and time preferences. Econ Theory 35, 19-36 (2008)

Roth, A.E.: Toward a focal-point theory of bargaining. In: Roth, A.E. (ed.) Game-Theoretic Models of Bargaining, chap. 12, Melbourne: Cambridge University Press (1985)

Roth, A.E.: Bargaining experiments. In: Kagel, J., Roth, A.E. (eds.) The Handbook of Experimental Economics, chap. 4, Princeton: Princeton University Press (1995)

Santamaria-Garcia, J.: Equilibrium selection in the Nash Demand Game: an evolutionary approach, working paper AD 2004-34, Universidad de Alicante (2004) 